\title{
An invariance principle for random traveling waves in one dimension
}

\author{
James Nolen*
}

September 21, 2010

\begin{abstract}
We consider solutions to a nonlinear reaction diffusion equation when the reaction term varies randomly with respect to the spatial coordinate. The nonlinearity is either the ignition nonlinearity or the bistable nonlinearity, under suitable restrictions on the size of the spatial fluctuations. It is known that the solution develops an interface which propagates with a well-defined speed in the large-time limit. The main result of this article is a functional central limit theorem for the random interface position.
\end{abstract}

\section{Introduction}

We consider solutions to a scalar reaction-diffusion equation in which the reaction term varies randomly with respect to the spatial coordinate:

$$
u_{t}=\Delta u+f(x, u, \omega), \quad x \in \mathbb{R} .
$$

When $f=f(u)$ is independent of $x$, there may be traveling wave solutions moving with constant speed. A similar phenomenon may occur even when there is spatial variation in $f$. When $f$ varies randomly with respect to $x$, in a statistically stationary way, such wave-like solutions may propagate with a well-defined asymptotic speed as $t \rightarrow \infty$, and in this setting it is natural to ask how the (random) solution fluctuates about its mean behavior. The main result of this article is a functional central limit theorem, or invariance principle, for the position of the interface in the large time limit. Under suitable scaling, the interface behaves like a Brownian motion with positive drift.

We suppose that $f$ has the form $f(x, u, \omega)=g(x, \omega) f_{0}(u)$ where $f_{0}(u)$ is a nonlinear, Lipschitz continuous function of $u$. We consider two cases for $f_{0}$. In both cases $f_{0}(0)=f_{0}(1)=0$, and the solution $u$ will take values in the interval $[0,1]$. In this first case, we suppose that

$$
f_{0}(u)=0 \text { for } u<\theta_{0}, f_{0}(u)>0 \text { for } u \in\left(\theta_{0}, 1\right), f(1)=0, f_{0}^{\prime}(1)<0,
$$

for some constant $\theta_{0} \in(0,1)$. Such a nonlinearity is sometimes called an ignition-type nonlinearity, and the constant $\theta_{0}$ is called the ignition temperature. The second case we consider is the bistable type nonlinearity:

$$
f_{0}(u)<0 \text { for } u \in\left(0, \theta_{0}\right), f_{0}(u)>0 \text { for } u \in\left(\theta_{0}, 1\right), f_{0}^{\prime}(0)<0, f_{0}^{\prime}(1)<0 .
$$

The function $g(x, \omega): \mathbb{R} \times \Omega \rightarrow(0, \infty)$ is a stationary, ergodic random field defined over a probability space $(\Omega, \mathcal{F}, \mathbb{P})$. We suppose that $g(x, \omega)$ is almost surely Lipschitz continuous with respect to $x$ and that for some constant $K_{g}$

$$
|g(x, \omega)-g(y, \omega)| \leq K_{g}|x-y|, \quad \forall x, y \in \mathbb{R}
$$

*Department of Mathematics, Duke University, Box 90320, Durham, NC 27708-0320, USA. (nolen@math.duke.edu). 
holds almost surely. Furthermore, we suppose that there are deterministic constants $g^{\min }, g^{\max }$ such that the bounds

$$
0<g^{\min } \leq g(x, \omega) \leq g^{\max }<\infty
$$

hold almost surely. For the bistable type nonlinearity, we make the additional assumption that

$$
\int_{0}^{1} \inf _{x}\left(g(x, \omega) f_{0}(u)\right) d u>\epsilon_{b}>0
$$

with probability one, for some positive constant $\epsilon_{b}$. This condition precludes the possibility of the front being pinned $(X(t)$ bounded for all $t)$, as described in [17], for example.

In the case that $f=f(u)$ is deterministic and independent of $x$, it has been known for many decades that semilinear reaction diffusion equations of the form

$$
u_{t}=u_{x x}+f(u)
$$

may admit stable traveling wave solutions existing for all $t \in \mathbb{R}$. For example, if $f$ is an ignitiontype or bistable-type nonlinearity, as described above, then $u \equiv 0$ and $u \equiv 1$ are equilibrium solutions to (1.7), and there exists a monotone traveling wave solution $u(t, x)=\phi(x-c t)$ for some unique speed $c>0$. The wave connects these two equilibria in the sense that $\lim _{x \rightarrow-\infty} u(t, x)=1$ and $\lim _{x \rightarrow+\infty} u(t, x)=0$, and the interface position $X(t)$ defined by $u(t, X(t))=\theta_{0}$ moves with constant speed. Moreover, the traveling wave is exponentially stable, which means that if $z(t, x)$ solves the Cauchy problem with suitable initial data at $t=0$, then

$$
\sup _{x \in \mathbb{R}}|u(t+\bar{\tau}, x)-z(t, x)| \leq C e^{-r t}, \quad \forall t>0
$$

for some constants $C, r>0$, and $\bar{\tau} \in \mathbb{R}$. Results on the existence and stability of traveling waves for various nonlinearities may be found in $[16,7,12,13,14,1,6,23]$.

\section{Generalized transition fronts}

It is natural to ask how solutions behave when the reaction term is no longer homogeneous with respect to the spatial coordinate:

$$
u_{t}=u_{x x}+f(x, u) .
$$

Is there a more general notion of traveling wave in this setting? If so, how do the spatial fluctuations in $f$ effect the wave and the interface? In this setting there have been extensions of the aforementioned results, even under very few structural conditions on the function $f$. If $f$ varies periodically with respect to $x$, then in many cases there exist solutions called pulsating traveling waves which oscillate periodically within the moving reference frame [25], [2], [30]. Even without periodicity in $x$, however, there may be nontrivial, wave-like solutions to (1.9) that exist for all $t \in \mathbb{R}$. These solutions are called generalized transition fronts or generalized traveling waves; this idea is due to H. Matano [18]. In the present setting, such a solution may be defined as follows. Suppose that $f(x, u)=g(x) f_{0}(u)$ where $f_{0}(u)$ is either the bistable or ignition nonlinearities. Then, under suitable assumptions about $g(x)$, there exist functions $u(t, x)$ satisfying the following:

(i) $u(t, x)$ solves $(1.9)$ for all $x \in \mathbb{R}$ and $t \in \mathbb{R}$.

(ii) $0<u<1$ and $u_{t}>0$, for all $x \in \mathbb{R}$ and $t \in \mathbb{R}$.

(iii) There is a continuous increasing function $X(t)$, the interface, such that $u(t, X(t))=\theta_{0}$, and

$$
\begin{aligned}
& \lim _{R \rightarrow \infty} \sup _{t \in \mathbb{R}} \sup _{x>R} u(t, x+X(t))=0, \\
& \lim _{R \rightarrow \infty} \inf _{t \in \mathbb{R}} \inf _{x<-R} u(t, x+X(t))=1 .
\end{aligned}
$$


The third property tells us that the width of the interface remains uniformly bounded in time. A function $u(t, x)$ satisfying these properties is called a generalized transition front or generalized traveling wave. The existence of such solutions to the equation (1.9) was proved in [20] and [21], while in [19] it was shown that the generalized transition front is unique and exponentially stable. Berestycki and Hamel [3] have developed a very general framework for such solutions to reaction-diffusion equations in inhomogeneous media. For some other recent work on generalized transition fronts we refer to $[4,24,31]$.

In the present article, we impose a particular statistical structure on $f$ and derive a central limit theorem for the interface $X(t, \omega)$ which is now a random variable. It was shown in [21] that there exists a generalized transition front solution $u(t, x, \omega): \mathbb{R}^{2} \times \Omega \rightarrow \mathbb{R}$ to (1.1) having the following properites:

(P1) For almost every $\omega \in \Omega, u(t, x, \omega)$ is a generlized transition front solution to (1.1).

(P2) The function $u(0, x, \omega): \mathbb{R} \times \Omega \rightarrow \mathbb{R}$ is jointly measureable with respect to the product field $\mathcal{B} \otimes \mathcal{F}$, where $\mathcal{B}$ is the Borel $\sigma$-algebra of $\mathbb{R}$.

(P3) There exists a measureable function $X(t, \omega): \mathbb{R} \times \Omega \rightarrow \mathbb{R}$ such that $u(t, X(t, \omega), \omega)=\theta_{0}$ and

$$
u(t, x+X(t, \omega), \omega)=u\left(0, x, \pi_{X(t, \omega)} \omega\right) .
$$

$\mathbb{P}$-almost surely.

(P4) There is a monotonically decreasing function $v(x)$ such that

$$
\begin{gathered}
0<v(x)<1 \text { and } v^{\prime}(x)<0 \text { for all } x \in \mathbb{R}, \quad v(+\infty)=0, v(-\infty)=1 \\
u(t, x+X(t, \omega), \omega) \leq v(x) \leq \theta_{0} e^{-\lambda x \quad} \quad \forall x>0, \quad t \in \mathbb{R} \\
u(t, x+X(t, \omega), \omega) \geq v(x) \quad \forall x<0, \quad t \in \mathbb{R} .
\end{gathered}
$$

Here $\lambda>0$ is a nonrandom constant independent of $t \in \mathbb{R}$ or $x \in \mathbb{R}$.

(P5) There are constants $C_{\min }, C_{\max }>0$ such that $C_{\min } \leq X^{\prime}(t, \omega) \leq C_{\max }$ holds for all $t$, $\mathbb{P}$-almost surely. For each $R>0$, there is a deterministic constant $\delta_{R}>0$ such that

$$
\inf _{t \in \mathbb{R}} \inf _{|x| \leq R} u_{t}(t, x+X(t, \omega), \omega) \geq \delta_{R}>0
$$

holds almost surely.

In property (P3), $\left\{\pi_{x}\right\}_{x \in \mathbb{R}}$ is a group of measure-preserving transformations which acts ergodically on $(\Omega, \mathcal{F}, \mathbb{P})$ such that $g(x+h, \omega)=g\left(x, \pi_{h} \omega\right)$. Therefore, property (P3) is analogous to the statement that $u(t, x)=\phi(x-c t)$ in the case that the medium is homogeneous. Property (P4) describes bounds on the wave speed and on the width of the interface that are uniform in time and uniform with respect to $\mathbb{P}$. This notion of a random transition front, or random traveling wave, is similar to Definition 2.2 of Shen [24].

In addition to proving existence of such a random traveling wave, the work of [21] shows that the interface position satisfies

$$
\lim _{t \rightarrow \infty} \frac{X(t, \omega)}{t}=c^{*}
$$

almost surely. So, the speed of the random traveling wave is well-defined in this asymptotic sense, and it is deterministic, due to the ergodicity assumption. This may be regarded as a law of large numbers for the interface position. In the case of the so-called KPP-type nonlinearity (e.g. $f(x, u, \omega)=g(x, \omega) f_{0}(u)$ with $f_{0}(u)=u(1-u)$ ), Freidlin and Gärtner [9, 8] used different techniques to obtain a similar asymptotic result for solutions to the Cauchy problem. In that case, a generalized traveling wave may not exist, yet the asymptotic speed of the interface is still well-defined as in (1.17). It is not known whether the invariance principle derived here can be extended to the KPP case. 


\section{The main results}

The main result of this paper is a functional central limit theorem, or invariance principle, for the randomly fluctuating interface $X(t, \omega)$. In addition to the aforementioned assumptions, we will assume that the random environment satisfies a mixing condition. To state this condition we define two families of $\sigma$-algebras $\left\{\mathcal{F}_{k}^{-}\right\}_{k \in \mathbb{Z}}$ and $\left\{\mathcal{F}_{k}^{+}\right\}_{k \in \mathbb{Z}}$ by

$$
\mathcal{F}_{k}^{-}=\sigma(g(x, \omega) \mid x \leq k) \quad \mathcal{F}_{k}^{+}=\sigma(g(x, \omega) \mid x \geq k) .
$$

These are the $\sigma$-algebras generated by the random fields $g(x, \omega) \chi_{(-\infty, k]}(x)$ and $g(x, \omega) \chi_{[k,+\infty]}(x)$, respectively. That is, $\mathcal{F}_{k}^{-} \subset \mathcal{F}$ is the smallest $\sigma$-algebra such that the random field $g(x, \omega) \chi_{(-\infty, k]}(x)$ : $\mathbb{R} \times \Omega \rightarrow \mathbb{R}$ is jointly $\mathcal{B} \otimes \mathcal{F}_{k}^{-}$-measureable. These families satisfy the order relations

$$
\mathcal{F}_{k}^{-} \subset \mathcal{F}_{k+1}^{-} \subset \mathcal{F}, \quad \text { and } \quad \mathcal{F} \supset \mathcal{F}_{k}^{+} \supset \mathcal{F}_{k+1}^{+}
$$

for all $k \in \mathbb{Z}$. Suppose that $\phi:[0, \infty) \rightarrow[0, \infty)$ is a continuous decreasing function such that $\phi(+\infty)=0$. We say that the random field $g(x, \omega)$ is $\phi$-mixing if the following holds: for all $j \geq k$ and any $\xi \in L^{2}\left(\Omega, \mathcal{F}_{k}^{-}, \mathbb{P}\right)$ and $\eta \in L^{2}\left(\Omega, \mathcal{F}_{j}^{+}, \mathbb{P}\right)$,

$$
|\mathbb{E}[\xi \eta]-\mathbb{E}[\xi] \mathbb{E}[\eta]| \leq \phi^{1 / 2}(j-k)\left(\mathbb{E}\left[\xi^{2}\right] \mathbb{E}\left[\eta^{2}\right]\right)^{1 / 2} .
$$

This mixing condition is a natural condition that arises in the proof of the central limit theorem for sums of stationary random variables (see [11]).

Theorem 1.1 Assume that the random field $g(x, \omega)$ is $\phi$-mixing with $\sum_{n=1}^{\infty}(\phi(n))^{1 / 2}<\infty$. Then either

(A.) there is a constant $\kappa>0$ such that

$$
\lim _{t \rightarrow \infty} \mathbb{P}\left(\frac{X(t, \omega)-t c^{*}}{\kappa \sqrt{t}}<\alpha\right)=F(\alpha), \quad \forall \alpha \in \mathbb{R}
$$

where $F(\alpha):=\frac{1}{\sqrt{2 \pi}} \int_{-\infty}^{\alpha} e^{-y^{2} / 2} d y$ is the cumulative normal distribution, or

$$
\lim _{t \rightarrow \infty} \mathbb{P}\left(\left|\frac{X(t, \omega)-t c^{*}}{\sqrt{t}}\right|>\alpha\right)=0
$$

for any $\alpha>0$.

Moreover, in Case A, the family of processes $\left\{Y_{n}(t)\right\}_{n \geq 0}$ defined by

$$
Y_{n}(t)=\frac{X(n t, \omega)-c^{*} n t}{\kappa \sqrt{n}}, \quad t \in[0,1]
$$

converges weakly to a standard Brownian motion on $[0,1]$ as $n \rightarrow \infty$.

The weak convergence described by the theorem means weak convergence of the family of probability measures induced by $\left\{Y_{n}(t)\right\}_{n}$ on the metric space $C([0,1])$, with the topology of uniform convergence. See [5] for more details about this notion of convergence. This theorem applies to the generalized transition front solution to (1.1), which is defined for all $t \in \mathbb{R}$. However, using the stability result of [19] we can extend this to solutions to the Cauchy problem for suitable initial data at $t=0$.

Corollary 1.2 Assume that the random field $g(x, \omega)$ is $\phi$-mixing with $\sum_{n=1}^{\infty} \phi^{1 / 2}(n)<\infty$. Let $z(t, x, \omega)$ satisfy (1.1) for $t \geq 0$ with initial condition $z(0, x, \omega)=z_{0}(x)$, where $z_{0}(x)$ satisfies

$$
0 \leq z_{0}(x) \leq 1, \quad \lim _{x \rightarrow-\infty} z_{0}(x)=1, \quad z_{0}(x) \leq C e^{-\alpha_{1} x}
$$

for some positive constants $C$ and $\alpha_{1}$. Let the interface position $X^{z}(t, \omega)$ be defined by

$$
X^{z}(t, \omega)=\sup \left\{x \in \mathbb{R} \mid z(t, x, \omega)=\theta_{0}\right\} .
$$


Then, if Case A holds,

$$
\lim _{t \rightarrow \infty} \mathbb{P}\left(\frac{X^{z}(t, \omega)-t c^{*}}{\kappa \sqrt{t}}<\alpha\right)=F(\alpha), \quad \forall \alpha \in \mathbb{R} .
$$

Moreover, the family of processes

$$
Y_{n}^{z}(t)=\frac{X^{z}(n t, \omega)-c^{*} n t}{\kappa \sqrt{n}}, \quad t \in[0,1]
$$

converges weakly to a standard Brownian motion on $[0,1]$ as $n \rightarrow \infty$. Otherwise, Case $B$ holds, and $\left(X^{z}(t, \omega)-t c^{*}\right) /(\sqrt{t}) \rightarrow 0$ in distribution, as $t \rightarrow \infty$.

Perhaps the most closely related asymptotic results for nonlinear PDEs with random coefficients are the works of Rezakhanlou [22], Wehr and Xin [27, 28], and Varadhan and Zygouras [29]. Rezakhanlou has studied fluctuations in solutions to stochastic Hamilton-Jacobi equations near a homogenization limit. Wehr and Xin have analyzed the large-time behavior of solutions to Burgers' equation with either random flux or random initial conditions. Varadhan and Zygouras have studied the asymptotic behavior of the tails of solutions to a semi-linear heat equation with a random source at the origin. Theorem 1.1 is also similar in spirit to the work of Kesten and Papanicolaou [15] who proved a CLT for a passive tracer transported by a random vector field. In that setting, a nonzero drift forces the particle to see new regions of the random environment, just as the assumptions (1.5) and (1.6) force the transition front to move forward into new regions.

\section{Strategy of the proof}

Let us describe the method of proof and the outline of the paper. To prove Theorem 1.1, we analyze the sequence of random variables $\left\{T_{n}\right\}_{n}$ which are the times at which the interface reaches the integer points $x=n \in \mathbb{Z}$. Because $X(t, \omega)$ is increasing in $t$, its inverse $T(x, \omega): \mathbb{R} \times \Omega \rightarrow \mathbb{R}$ is well-defined by the relation $x=X(T(x, \omega), \omega)$. Thus,

$$
T_{n}=T(n, \omega), \quad n=0,1,2, \ldots
$$

From the definition of $T$ and $u$ it follows that $u(x+y, T(y, \omega), \omega)=u\left(x, 0, \pi_{y} \omega\right)$ for all $y \in \mathbb{R}$. This means that as the wave passes through the point $y$ the statistics of the profile of the wave are invariant with respect to $y$. Consequently, the increments $\Delta T_{n}=T_{n+1}-T_{n}$ are stationary, and $T_{n}$ satisfies a law of large numbers [21]:

$$
\lim _{n \rightarrow \infty} \frac{T_{n}}{n}=\lim _{n \rightarrow \infty} \frac{1}{n} \sum_{j=0}^{n-1} \Delta T_{j}=\bar{\tau}=\left(c^{*}\right)^{-1},
$$

which is another way of expressing (1.17). We will show that the limit

$$
\lim _{n \rightarrow \infty} \mathbb{E}\left[\left|\frac{T_{n}-n \bar{\tau}}{\sqrt{n}}\right|^{2}\right]=\sigma^{2}
$$

exits for some constant $\sigma^{2} \geq 0$, and that if $\sigma^{2}>0$, then the family of processes

$$
Z_{n}(x)=\frac{T_{x n}-n x \bar{\tau}}{\sigma \sqrt{n}}, \quad x \geq 0 .
$$

converges weakly to the standard Brownian motion on $[0, R]$ for any $R>0$. From this Theorem 1.1 will follow, with $\kappa=\left(c^{*}\right)^{3 / 2} \sigma$.

The increments $\Delta T_{n}$ are correlated in a complicated way through the nonlinear equation (1.1); this is the fundamental obstacle to deriving the main result. Nevertheless, we may derive (1.26) and (1.27) using the martingale approximation method due to Gordin [10] and developed 
further in [11] and [26]. In order to make this approximation strategy work, one must estimate the conditional expectations $\mathbb{E}\left[\Delta T_{k} \mid \mathcal{F}_{j}^{-}\right]$and $\mathbb{E}\left[\Delta T_{j} \mid \mathcal{F}_{k}^{+}\right]$when $k$ is much larger than $j$. If $\Delta T_{k}$

were $\mathcal{F}_{r}^{+}$-measureable for some $r$ with $j<r<k$, then we could bound $\mathbb{E}\left[\Delta T_{k} \mid \mathcal{F}_{j}^{-}\right]$using the mixing assumption (1.19). However, the mixing condition (1.19) is imposed on the environment, rather than on the $\sigma$-algebras generated by the interface position $X(t, \omega)$ or by the increments $\Delta T_{n}(\omega)$. In fact, $\Delta T_{k}$ is not $\mathcal{F}_{r}^{+}$-measureable for any $r \in \mathbb{R}$, and in the case of the bistable-type nonlinearity, $\Delta T_{k}$ is not $\mathcal{F}_{r}^{-}$-measureable for any $r \in \mathbb{R}$. Therefore, a key step in applying the martingale approximation argument will be a stability estimate for perturbations of generalized transition fronts. One may interpret these estimates to mean that the movement of the interface depends primarily on the local environment, and only weakly on the distant past and distant future. In other words, the stability of the generalized transition front implies that the interface "forgets its past"; this fact leads to the universal Gaussian behavior of the interface position in the large time limit.

The paper is organized as follows. In Section 2 we first recall a general sufficient condition for the central limit theorem to hold for sums of identically distributed random variables. This is Theorem 2.1. We then apply Theorem 2.1 to derive (1.26) and the invariance principle for $Z_{n}(x)$ defined by (1.27). From this and the inverse relationship between $T_{n}(\omega)$ and $X(t, \omega)$ we then derive the main results, Theorem 1.1 and Corrolary 1.2. Our application of Theorem 2.1 will rely on some crucial estimates on the conditional expectations $\mathbb{E}\left[\Delta T_{k} \mid \mathcal{F}_{j}^{-}\right]$and $\mathbb{E}\left[\Delta T_{k} \mid \mathcal{F}_{j}^{+}\right]$, which we state in Proposition 2.2. Sections $3-6$ are devoted to proving Proposition 2.2. In particular, Section 3 establishes the important connection between the stability of the traveling front solution and the key estimates on $\mathbb{E}\left[\Delta T_{k} \mid \mathcal{F}_{j}^{-}\right]$and $\mathbb{E}\left[\Delta T_{k} \mid \mathcal{F}_{j}^{+}\right]$. In Section 7 , we construct an example demonstrating that Case A may hold.

\section{Acknowledgements}

This work was partially supported by a postdoctoral fellowship from the NSF. I am grateful for the hospitality of the math department at Stanford University where much of this work was completed. I also thank the referees for careful reading and helpful comments.

\section{Proof of the invariance principle}

\subsection{A general central limit theorem and related estimates}

We first prove (1.26) and the invariance principle for $Z_{n}(x)$ and then show how this leads to Theorem 1.1, the invariance principle for for $X(t, \omega)$. We will apply the following general result of Hall and Heyde [11] (see Section 5.4 therein, as well as Volný [26]), which yields the invariance principle for stationary sequences that can be approximated suitably by sequences of martingale differences:

Theorem 2.1 (See Hall and Heyde [11], and Volný [26]) Suppose that a sequence $\left\{\eta_{k}\right\}_{k} \subset$ $L^{2}(\Omega, \mathcal{F}, \mathbb{P})$ satisfies $\mathbb{E}\left[\eta_{k}\right]=0, \eta_{k+j}(\omega)=\eta_{k}\left(\pi_{j} \omega\right)$ for all $j, k \in \mathbb{Z}$, and that the two series

$$
\sum_{k=1}^{\infty} \mathbb{E}\left[\eta_{k} \mid \mathcal{F}_{0}^{-}\right] \quad \text { and } \quad \sum_{k=1}^{\infty}\left(\eta_{0}-\mathbb{E}\left[\eta_{0} \mid \mathcal{F}_{k}^{-}\right]\right)
$$

converges in $L^{2}(\Omega, \mathcal{F}, \mathbb{P})$. Then, the limit

$$
\sigma^{2}=\lim _{N \rightarrow \infty} \frac{1}{N} \mathbb{E}\left[\left|\sum_{k=0}^{N-1} \eta_{k}\right|^{2}\right]<\infty
$$

exists. If $\sigma^{2}>0$ and $S_{k}=\sum_{j=0}^{k-1} \eta_{j}$, the family of processes

$$
\bar{Z}_{n}(x)=\frac{1}{\sigma \sqrt{n}}\left(S_{k}+(n x-k) \eta_{k}\right), \quad k \leq n x \leq k+1, \quad k=0,1, \ldots, n-1
$$


converge weakly to a standard Brownian motion on $[0,1]$, in the sense of $C([0,1])$ with the topology of uniform convergence.

Since $\mathbb{E}\left[\Delta T_{k}\right]=\bar{\tau}>0$, we define the centered random variables $\Delta \tilde{T}_{k}=\Delta T_{k}-\bar{\tau}$, and we now demonstrate that the random variables $\eta_{k}=\Delta \tilde{T}_{k}$ satisfy the criteria of this theorem. By definition, $\mathbb{E}\left[\Delta \tilde{T}_{k}\right]=0$. The stationarity of the increments $\Delta \tilde{T}_{k}$ follows from the definition of $T(x, \omega)$ and property (1.12) of $u$. That is, since $u\left(T_{k+j}, x+k+j, \omega\right)=u\left(0, x, \pi_{k+j} \omega\right)$ for all $k, j \in \mathbb{Z}, \Delta T_{k+j}(\omega)=\Delta T_{0}\left(\pi_{k+j} \omega\right)=\Delta T_{0}\left(\pi_{k} \pi_{j} \omega\right)=\Delta T_{k}\left(\pi_{j} \omega\right)$. Therefore, our main task is to demonstrate that $(2.1)$ holds for $\eta_{k}=\Delta \tilde{T}_{k}$, which means that the two series

$$
\sum_{k=1}^{\infty} \mathbb{E}\left[\Delta \tilde{T}_{k} \mid \mathcal{F}_{0}^{-}\right] \quad \text { and } \quad \sum_{k=1}^{\infty}\left(\Delta \tilde{T}_{0}-\mathbb{E}\left[\Delta \tilde{T}_{0} \mid \mathcal{F}_{k}^{-}\right]\right)
$$

converge in $L^{2}(\Omega, \mathcal{F}, \mathbb{P})$. We now state some crucial estimates of $\mathbb{E}\left[\Delta \tilde{T}_{k} \mid \mathcal{F}_{j}^{-}\right]$and $\mathbb{E}\left[\Delta \tilde{T}_{k} \mid \mathcal{F}_{j}^{+}\right]$that will enable us to verify the convergence of these series.

Proposition 2.2 Let $f_{0}(u)$ be either the ignition-type or bistable nonlinearity. There are constants $C_{1}, C_{2}>0$ such that

$$
\left\|\Delta \tilde{T}_{k}-\mathbb{E}\left[\Delta \tilde{T}_{k} \mid \mathcal{F}_{j}^{+}\right]\right\|_{L^{2}(\Omega, \mathcal{F}, \mathbb{P})} \leq C_{1} e^{-C_{2}(k-j)}
$$

holds for all $k \geq j$, and

$$
\left\|\Delta \tilde{T}_{k}-\mathbb{E}\left[\Delta \tilde{T}_{k} \mid \mathcal{F}_{j}^{-}\right]\right\|_{L^{2}(\Omega, \mathcal{F}, \mathbb{P})} \leq C_{1} e^{-C_{2}(j-k)}
$$

holds for all $j \geq k$. The constants $C_{1}$ and $C_{2}$ do not depend on the function $\phi$ in (1.19).

We postpone the proof of Proposition 2.2 until Section 3, and we continue verifying the convergence of the series in (2.4). The convergence of the second series in (2.4) follows immediately from (2.6). For the first series, we need to estimate the conditional expectation $\mathbb{E}\left[\Delta \tilde{T}_{k} \mid \mathcal{F}_{0}^{-}\right]$and show that

$$
\lim _{m \rightarrow \infty} \limsup _{n \rightarrow \infty} \mathbb{E}\left[\left(\sum_{k=m}^{n} \mathbb{E}\left[\Delta \tilde{T}_{k} \mid \mathcal{F}_{0}^{-}\right]\right)^{2}\right]=0 .
$$

This will follow from (2.5) and the mixing condition (1.19), as we now demonstrate.

Let $\theta \in L^{2}\left(\Omega, \mathcal{F}_{0}^{-}, \mathbb{P}\right)$ with $\mathbb{E}\left(\theta^{2}\right)=1$. For any integer $1 \leq j<k$,

$$
\mathbb{E}\left(\theta \mathbb{E}\left[\Delta \tilde{T}_{k} \mid \mathcal{F}_{0}^{-}\right]\right)=\mathbb{E}\left(\theta \Delta \tilde{T}_{k}\right)=\mathbb{E}\left(\theta \mathbb{E}\left[\Delta \tilde{T}_{k} \mid \mathcal{F}_{j}^{+}\right]\right)+\mathbb{E}\left(\theta\left(\Delta \tilde{T}_{k}-\mathbb{E}\left[\Delta \tilde{T}_{k} \mid \mathcal{F}_{j}^{+}\right]\right)\right)
$$

We bound the first term on the right by choosing $j=\lfloor k / 2\rfloor$ and using the mixing condition (1.19). Since $\mathbb{E}\left[\Delta \tilde{T}_{k}\right]=0$, we conclude

$$
\left|\mathbb{E}\left(\theta \mathbb{E}\left[\Delta \tilde{T}_{k} \mid \mathcal{F}_{j}^{+}\right]\right)\right| \leq \phi^{1 / 2}(j-1) \mathbb{E}\left[(\Delta \tilde{T})^{2}\right]^{1 / 2} \leq C \phi^{1 / 2}(\lfloor k / 2\rfloor-1)
$$

Here we have used the fact that $0 \leq \Delta T \leq\left(C_{\text {min }}\right)^{-1}$. We estimate the second term in $(2.8)$ with the Cauchy-Schwarz inequality and (2.5):

$$
\left|\mathbb{E}\left(\theta\left(\Delta \tilde{T}_{k}-\mathbb{E}\left[\Delta \tilde{T}_{k} \mid \mathcal{F}_{j}^{+}\right]\right)\right)\right| \leq \mathbb{E}\left(\theta^{2}\right)^{1 / 2}\left\|\Delta \tilde{T}_{k}-\mathbb{E}\left[\Delta \tilde{T}_{k} \mid \mathcal{F}_{j}^{+}\right]\right\|_{L^{2}(\Omega, \mathcal{F}, \mathbb{P})} \leq C_{1} e^{-C_{2} k / 2}
$$

Since $\theta \in L^{2}\left(\Omega, \mathcal{F}_{0}^{-}, \mathbb{P}\right)$ was arbitrary, we conclude that

$$
\mathbb{E}\left(\left|\mathbb{E}\left[\Delta \tilde{T}_{k} \mid \mathcal{F}_{0}^{-}\right]\right|^{2}\right)^{1 / 2} \leq C_{1} e^{-C_{2} k / 2}+C \phi^{1 / 2}(\lfloor k / 2\rfloor-1)
$$


Therefore, the triangle inequality implies

$$
\begin{aligned}
\mathbb{E}\left[\left(\sum_{k=m}^{n} \mathbb{E}\left[\Delta \tilde{T}_{k} \mid \mathcal{F}_{0}^{-}\right]\right)^{2}\right]^{1 / 2} & \leq \sum_{k=m}^{n} \mathbb{E}\left[\left(\mathbb{E}\left[\Delta \tilde{T}_{k} \mid \mathcal{F}_{0}^{-}\right]\right)^{2}\right]^{1 / 2} \\
& \leq \sum_{k=m}^{n}\left(C_{1} e^{-C_{2} k / 2}+C \phi^{1 / 2}(\lfloor k / 2\rfloor-1)\right)
\end{aligned}
$$

Since the series $\sum_{n=1}^{\infty} \phi^{1 / 2}(n)$ converges, it follows that $\sum_{k=1}^{\infty} \mathbb{E}\left[\Delta \tilde{T}_{k} \mid \mathcal{F}_{0}^{-}\right]$converges.

Now, having shown that both series in $(2.4)$ converge in $L^{2}(\Omega, \mathcal{F}, \mathbb{P})$, we may apply Theorem 2.1 with $\eta_{k}=\Delta \tilde{T}_{k}$. Then, if $\sigma^{2}>0$, the process

$$
\bar{Z}_{n}(x)=\frac{1}{\sigma \sqrt{n}}\left(T_{k}-\bar{\tau} k+(n x-k) \Delta \tilde{T}_{k}\right), \quad k \leq n x \leq k+1, \quad k=0,1, \ldots, n-1 .
$$

converges weakly to the standard Brownian motion $W(x)$. In particular, for any $0 \leq x_{1} \leq \cdots \leq$ $x_{k}$, the finite dimensional distributions of $\bar{Z}_{n}\left(x_{1}\right), \ldots, \bar{Z}_{n}\left(x_{k}\right)$ converge to those of $W\left(x_{1}\right), \ldots, W\left(x_{k}\right)$ as $n \rightarrow \infty$, and the family of measures on $C([0,1])$ induced by the processes $\left\{\bar{Z}_{n}(x)\right\}_{n}$ is tight. In the space $C([0,1])$ tightness is equivalent to these two conditions:

(T1) For any $\epsilon_{1}, \epsilon_{2}>0$, there is $\delta \in(0,1)$ and $N \in \mathbb{Z}$ such that

$$
\mathbb{P}\left(\sup _{\substack{s, t \in[0,1] \\|t-s| \leq \delta}}\left|\bar{Z}_{n}(t)-\bar{Z}_{n}(s)\right| \geq \epsilon_{1}\right) \leq \epsilon_{2}
$$

for all $n \geq N$.

(T2) For any $\epsilon_{2}>0$,

$$
\mathbb{P}\left(\left|Y_{n}(0)\right| \geq \beta\right) \leq \epsilon_{2}, \quad \forall n \geq 1
$$

holds if $\beta$ is sufficiently large.

See Theorem 8.2 of [5] for a proof of this equivalence.

The invariance principle for $\bar{Z}_{n}(x)$ implies an invariance principle for $Z_{n}(x)$ defined by (1.27), as well. To see this, suppose $k \in \mathbb{Z}, k \leq n$, and $|n x-k| \leq 1$. Then

$$
Z_{n}(x)=\frac{T_{k}-\bar{\tau} k}{\sigma \sqrt{n}}+\frac{\left(T_{n x}-T_{k}\right)+\bar{\tau}(k-n x)}{\sigma \sqrt{n}}
$$

Therefore, since $\bar{Z}_{n}(x)=Z_{n}(x)$, if $n x \in \mathbb{Z}$, and since $0<C_{\min } \leq X^{\prime} \leq C_{\max }$ (Property P5), it follows that

$$
\sup _{x \in[0,1]}\left|Z_{n}(x)-\bar{Z}_{n}(x)\right| \leq \frac{\left(C_{m i n}\right)^{-1}+\bar{\tau}}{\sigma \sqrt{n}}
$$

Therefore, for any $\epsilon>0$,

$$
\lim _{n \rightarrow \infty} \mathbb{P}\left(\sup _{x \in[0,1]}\left|Z_{n}(x)-\bar{Z}_{n}(x)\right|>\epsilon\right)=0 .
$$

Therefore, $Z_{n}(x) \rightarrow W(x)$ weakly as $n \rightarrow \infty$, in the sense of $C([0,1])$. 


\subsection{Proof of the invariance principle for $X(t)$}

Now we will use the weak convergence of the processes $\left\{Z_{n}(x)\right\}_{n}$ to derive an invariance principle for the interface position $X(t, \omega)$, establishing Theorem 1.1. Suppose $\sigma^{2}>0$ and that $\kappa=$ $\left(c^{*}\right)^{3 / 2} \sigma$. Define the family of continuous processes

$$
Y_{n}(t)=\frac{X(n t)-c^{*} n t}{\kappa \sqrt{n}}, \quad t \geq 0
$$

By Theorem 8.1 of [5], it suffices to show that

(i) For any $0 \leq t_{1} \leq \cdots \leq t_{K} \leq 1$, the finite dimensional distributions of $\left(Y_{n}\left(t_{1}\right), \ldots, Y_{n}\left(t_{K}\right)\right)$ converge to those of $W\left(t_{1}\right), \ldots, W\left(t_{K}\right)$ as $n \rightarrow \infty$.

(ii) The family of measures on $C([0,1])$ induced by $\left\{Y_{n}(t)\right\}_{n}$ is tight, meaning that conditions (T1) and (T2) hold for $\left\{Y_{n}(t)\right\}_{n}$.

\section{Convergence of the finite dimensional distributions}

Let $\left\{t_{k}\right\}_{k=1}^{K}$ be a finite subset of $[0,1]$, and let $\left\{\alpha_{k}\right\}_{k=1}^{K} \subset \mathbb{R}$. By definition of $X$ and $T$, $X\left(T_{x}(\omega), \omega\right)=x$ for all $x \in \mathbb{R}$, with probability one. Therefore

$$
\begin{aligned}
\mathbb{P}\left(Y_{n}\left(t_{k}\right)<\alpha_{k}, \forall k=1, \ldots, K\right) & =\mathbb{P}\left(X\left(n t_{k}\right)<\sqrt{n} \kappa \alpha_{k}+c^{*} n t_{k}, \forall k\right) \\
& =\mathbb{P}\left(T_{\sqrt{n} \kappa \alpha_{k}+c^{*} n t_{k}}>n t_{k}, \forall k\right) \\
& =\mathbb{P}\left(Z_{n}\left(x_{k}^{n}\right)>-\frac{\bar{\tau} \kappa \alpha_{k}}{\sigma}, \forall k\right)
\end{aligned}
$$

where we have defined $x_{k}^{n}=n^{-1 / 2} \kappa \alpha_{k}+c^{*} t_{k}$, for $k=1, \ldots, K$. Now for any $\epsilon>0$, we have

$$
\begin{aligned}
\mathbb{P}\left(Y_{n}\left(t_{k}\right)<\alpha_{k}, \forall k\right)= & \mathbb{P}\left(Z_{n}\left(x_{k}^{n}\right)>\frac{-\bar{\tau} \kappa \alpha_{k}}{\sigma}, \forall k\right) \\
\leq & \mathbb{P}\left(Z_{n}\left(c^{*} t_{k}\right)>\frac{-\bar{\tau} \kappa \alpha_{k}}{\sigma}-\epsilon, \quad \forall k\right) \\
& +\mathbb{P}\left(Z_{n}\left(c^{*} t_{k}\right)-Z_{n}\left(x_{k}^{n}\right)<-\epsilon, \quad \forall k\right) .
\end{aligned}
$$

Since $Z_{n}$ converges weakly to $W$, in the sense of $C([0, R])$ for any $R>0$, the associated family of measures is tight and therefore

$$
\begin{aligned}
\limsup _{n \rightarrow \infty} \mathbb{P} & \left(Z_{n}\left(c^{*} t_{k}\right)-Z_{n}\left(x_{k}^{n}\right)<-\epsilon, \quad \forall k\right) \\
& \leq \limsup _{n \rightarrow \infty} \mathbb{P}\left(\sup _{\substack{x, y \in\left[0, c^{*}+1\right] \\
|x-y| \leq n^{-1 / 2} \kappa \alpha_{k}}}\left|Z_{n}(x)-Z_{n}(y)\right|>\epsilon\right)=0 .
\end{aligned}
$$

So, we see that for any $\epsilon>0$,

$$
\limsup _{n \rightarrow \infty} \mathbb{P}\left(Y_{n}\left(t_{k}\right)<\alpha_{k}, \quad \forall k\right) \leq \prod_{k=1}^{K}\left(1-F\left(\frac{-\bar{\tau} \kappa \alpha_{k} \sigma^{-1}-\epsilon}{\sqrt{c^{*} t_{k}}}\right)\right)
$$

where $F$ is the cumulative normal distribution. Similarly,

$$
\begin{aligned}
\mathbb{P}\left(Y_{n}\left(t_{k}\right)<\alpha_{k}, \forall k\right) \geq & \mathbb{P}\left(Z_{n}\left(c^{*} t_{k}\right)>\frac{-\bar{\tau} \kappa \alpha_{k}}{\sigma}+\epsilon, \quad \forall k\right) \\
& -\mathbb{P}\left(Z_{n}\left(c^{*} t_{k}\right)-Z_{n}\left(x_{k}^{n}\right)>\epsilon, \quad \forall k\right) .
\end{aligned}
$$

Therefore,

$$
\liminf _{n \rightarrow \infty} \mathbb{P}\left(Y_{n}\left(t_{k}\right)<\alpha_{k}, \quad \forall k\right) \geq \prod_{k=1}^{K}\left(1-F\left(\frac{-\bar{\tau} \kappa \alpha_{k} \sigma^{-1}+\epsilon}{\sqrt{c^{*} t_{k}}}\right)\right)
$$


Recall that $\kappa=\left(c^{*}\right)^{3 / 2} \sigma$ and $\bar{\tau}=\left(c^{*}\right)^{-1}$. Since $\epsilon$ is arbitrary, we conclude that

$$
\lim _{n \rightarrow \infty} \mathbb{P}\left(Y_{n}\left(t_{k}\right)<\alpha_{k}, \quad \forall k\right)=\prod_{k=1}^{K}\left(1-F\left(\frac{-\bar{\tau} \kappa \alpha_{k}}{\sigma \sqrt{c^{*} t_{k}}}\right)\right)=\prod_{k=1}^{K} F\left(\frac{\alpha_{k}}{\sqrt{t_{k}}}\right),
$$

which is equal to $\mathbb{P}\left(W\left(t_{k}\right) \leq \alpha_{k}, \quad \forall k=1, \ldots, K\right)$.

\section{Tightness}

The tightness of the family of measures induced by $\left\{Y_{n}(t)\right\}_{n}$ also follows from the weak convergence of $Z_{n}(x)$. Verifying condition (T2) for $\left\{Y_{n}(t)\right\}_{n}$ is trivial since $Y_{n}(0)=0$, almost surely. Now we need to verify condition (T1) for $\left\{Y_{n}(t)\right\}_{n}$. Observe that $X(n t)-X(n s)-n c^{*}(t-s)>$ $\kappa \sqrt{n} \epsilon$ if and only if

$$
T_{X(n s)+\kappa \sqrt{n} \epsilon+c^{*} n(t-s)}-T_{X(n s)}<n(t-s) .
$$

So, if we define $x_{s}^{n}=n^{-1} X(n s)$, then for any $\epsilon>0$

$$
\begin{aligned}
\mathbb{P}\left(\sup _{\substack{s, t \in[0,1] \\
s-t \leq \delta}} Y_{n}(s)-Y_{n}(t) \geq \epsilon\right) & =\mathbb{P}\left(\sup _{\substack{s, t \in[0,1] \\
s-t \leq \delta}} T_{n\left(x_{s}^{n}+\kappa n^{-1 / 2} \epsilon+c^{*}(t-s)\right)}-T_{n x_{s}^{n}}-n(t-s)<0\right) \\
& \leq \mathbb{P}\left(\inf _{\substack{x \in\left[0, C_{\text {max }}\right] \\
r \leq \delta}} Z_{n}\left(x+\kappa n^{-1 / 2} \epsilon+c^{*} r\right)-Z_{n}(x)<-\frac{\bar{\tau} \kappa \epsilon}{\sigma}\right) .
\end{aligned}
$$

Therefore, the tightness condition (T1) for $\left\{Z_{n}(t)\right\}_{n}$ implies that

$$
\lim _{\delta \rightarrow 0} \lim _{n \rightarrow \infty} \mathbb{P}\left(\sup _{\substack{s, t \in[0,1] \\ s-t \leq \delta}} Y_{n}(s)-Y_{n}(t) \geq \epsilon\right)=0 .
$$

Similarly, we find that

$$
\begin{aligned}
\mathbb{P}\left(\inf _{\substack{s, t \in[0,1] \\
s-t \leq \delta}} Y_{n}(s)-Y_{n}(t) \leq-\epsilon\right) & =\mathbb{P}\left(\inf _{\substack{s, t \in[0,1] \\
s-t \leq \delta}} T_{n\left(x_{s}^{n}-\kappa n^{-1 / 2} \epsilon+c^{*}(t-s)\right)}-T_{n x_{s}^{n}}-n(t-s)>0\right) \\
& \leq \mathbb{P}\left(\sup _{\substack{x \in\left[0, C_{\max }\right] \\
r \leq \delta}} Z_{n}\left(x-\kappa n^{-1 / 2} \epsilon+c^{*} r\right)-Z_{n}(x)>\frac{\bar{\tau} \kappa \epsilon}{\sigma}\right) .
\end{aligned}
$$

So, we conclude that

$$
\lim _{\delta \rightarrow 0} \lim _{n \rightarrow \infty} \mathbb{P}\left(\inf _{\substack{s, t \in[0,1] \\ s-t \leq \delta}} Y_{n}(s)-Y_{n}(t) \leq-\epsilon\right)=0,
$$

holds as well. The combination of (2.17) and (2.18) shows that condition (T1) holds for $\left\{Y_{n}(t)\right\}_{n}$. This completes the proof of Theorem 1.1.

\subsection{Proof of Corollary 1.2}

Having established Theorem 1.1, we now prove Corollary 1.2, the invariance principle for $X^{z}(t, \omega)$. From [19] we know that

$$
\sup _{x \in \mathbb{R}}|z(t, x, \omega)-u(t+\tau, x, \omega)| \leq C e^{-r t}
$$


holds, $\mathbb{P}$-almost surely, where $\tau=\tau(\omega)$ is a random shift satisfying $|\tau(\omega)| \leq C_{\tau}$ and $r>0$. This and the fact that $\left|X^{\prime}(t, \omega)\right| \leq C_{\max }$ imply that $\left\|X^{z}(t, \omega)-X(t, \omega)\right\|_{L^{\infty}(\Omega)}$ is uniformly bounded for $t \geq 0$. Therefore,

$$
\lim _{t \rightarrow \infty} \mathbb{P}\left(\frac{X^{z}(t)-t c^{*}}{\sqrt{t}}<\beta\right)=\lim _{t \rightarrow \infty} \mathbb{P}\left(\frac{X(t)-t c^{*}}{\sqrt{t}}<\beta-\frac{X^{z}(t)-X(t)}{\sqrt{t}}\right) .
$$

In Case $\mathrm{A}, \kappa^{2}>0$, and this limit is $F(\beta / \kappa)$. Otherwise, $\kappa^{2}=0$ and the limit is equal to 0 for $\beta<0$, and it is equal to 1 for $\beta>0$. In Case $A$,

$$
\lim _{n \rightarrow \infty} \mathbb{P}\left(\sup _{t \in[0,1]}\left|Y_{n}(t)-Y_{n}^{z}(t)\right|>\epsilon\right)=\lim _{n \rightarrow \infty} \mathbb{P}\left(\sup _{t \in[0,1]}\left|\frac{X^{z}(n t)-X(n t)}{\kappa \sqrt{n}}\right|>\epsilon\right)=0
$$

for any $\epsilon>0$. Therefore, $Y_{n}^{z}(x) \rightarrow W(x)$ weakly as $n \rightarrow \infty$, in the sense of $C([0,1])$.

\section{Uniform Stability Estimates}

The goal of this section is to prove the estimates in Proposition 2.2. Our strategy will be to use the stability of the traveling wave to show that the movement of the interface depends primarily on the local environment and is not strongly effected by the distant past and distant future. This is the essence of Theorems 3.1 and 3.4, which we prove later in Section 4 and Section 5, respectively.

\section{Dependence on the past}

We first examine the dependence of $\Delta T_{k}$ on $g(x, \omega)$ for $x \ll k$, which is the environment that lies far behind the interface. Our idea is to modify the equation for $u$ in the region $x<0$ and then consider how the solution to the modified equation compares with the solution to the original unmodified equation at large times. Suppose that $f_{0}(u)$ is either the ignition-type nonlinearity or the bistable type nonlinearity. Let $\hat{g}(x, \omega)$ be a jointly $\mathcal{B} \otimes \mathcal{F}_{0}^{+}$-measureable random field which satisfies (1.4) and (1.5) and which agrees with $g(x, \omega)$ for $x>0$, almost surely. For example, we might let $\hat{g}(x, \omega)$ be defined by

$$
\hat{g}(x, \omega)= \begin{cases}g(x, \omega), & x \geq 0 \\ g(0, \omega), & x \leq 0\end{cases}
$$

Roughly speaking, the following theorem implies that the wave "forgets the past" rapidly, since the solution to the modified equation associated with the modified field $\hat{g}$ converges rapidly to the transition front which solves the original unmodified equation:

Theorem 3.1 Let $z(t, x, \omega)$ solve the modified initial value problem

$$
z_{t}=z_{x x}+\hat{g}(x, \omega) f_{0}(z), \quad x \in \mathbb{R}, \quad t \geq 0
$$

with deterministic initial condition $z(0, x, \omega)=z_{0}(x)$ satisfying

$$
0 \leq z_{0}(x) \leq 1, \quad \lim _{x \rightarrow-\infty} z_{0}(x)=1, \quad z_{0}(x) \leq K e^{-\alpha_{1} x}
$$

for some $K, \alpha_{1}>0$. There are constants $C, C_{\tau}, r>0$ and a random variable $\tau(\omega)$ such that, almost surely with respect to $\mathbb{P}$, both $|\tau(\omega)| \leq C_{\tau}$ and

$$
\sup _{x \in \mathbb{R}}|z(t, x, \omega)-u(t+\tau(\omega), x, \omega)| \leq C e^{-r t}
$$

hold for all $t>0$. 
If $z(t, x, \omega)$ solves the modified problem $(3.1)$, we let $T_{k}^{z}(\omega)$ denote the time at which the interface corresponding to $z(t, x, \omega)$ reaches the point $x=k$ :

$$
T_{k}^{z}(\omega)=\inf \left\{s \geq 0 \mid z(s, k, \omega)=\theta_{0}\right\} .
$$

Due to the definition of $\hat{g}$ and $z$, the increments $\Delta T_{k}^{z}=T_{k+1}^{z}-T_{k}^{z}$ are $\mathcal{F}_{0}^{+}$-measureable random variables, while $\Delta T_{k}$ is not $\mathcal{F}_{j}^{+}$-measureable for any $j$. However, using Theorem 3.1 we derive a uniform estimate of the difference between $\Delta T_{k}^{z}$ and $\Delta T_{k}$, from which we will obtain (2.5):

Corollary 3.2 There are deterministic constants $C, h>0$ such that

$$
\left\|\Delta T_{k}^{z}(\omega)-\Delta T_{k}\right\|_{L^{\infty}(\Omega, \mathcal{F}, \mathbb{P})} \leq C e^{-h k}
$$

for all $k \geq 0$.

We will prove Corollary 3.2 in Section 6.

\section{Dependence on the future}

Next, we examine the dependence of $\Delta T_{k}$ on $g(x)$ for $x \gg k$, which is the environment far ahead of the interface. We will treat separately the two cases of the nonlinearity $f_{0}(u)$.

Lemma 3.3 Let $f_{0}(u)$ be the ignition-type nonlinearity. For all integers $k \in \mathbb{Z}$, the random variable $T_{k}$ is $\mathcal{F}_{k}^{-}$-measureable. Consequently, $\Delta T_{k}=\mathbb{E}\left[\Delta T_{k} \mid \mathcal{F}_{N}^{-}\right]$for any $N \geq k+1$.

Proof: The fact that $T_{k}$ is $\mathcal{F}_{k}^{-}$-measureable follows from the construction of the traveling wave in [21] and the fact that $f_{0}(u)$ vanishes where $u \leq \theta_{0}$. The traveling wave satisfies $u(t, x, \omega) \leq \theta_{0}$ for all $x>k$ and $t<T_{k}(\omega)$, so that $f_{0}(u)=0$ for all $x>k$ and $t<T_{k}(\omega)$. Therefore,

$$
u_{t}=u_{x x}+g(x, \omega) \chi_{(-\infty, k]}(x) f_{0}(u) \quad x \in \mathbb{R}, \quad t<T_{k}(\omega)
$$

holds $\mathbb{P}$-almost surely.

Observe that for the ignition-type nonlinearity, (2.6) is an immediate consequence of Lemma 3.3. If $f_{0}(u)$ is the bistable-type nonlinearity, however, $f_{0}$ does not vanish on an open set containing $u=0$, and in this case more analysis is required to estimate $\Delta T_{k}-\mathbb{E}\left[\Delta T_{k} \mid \mathcal{F}_{N}^{-}\right]$. To this end, we will consider solutions to a modified equation, which has been modified only for $x \gg 1$; comparing the solution to the modified equation with the solution to the unmodified equation will show that $u$ does not strongly depend on $g(x, \omega)$ for $x \gg 1$. We let $\hat{g}_{N}(x, \omega)$ be a jointly $\mathcal{B} \otimes \mathcal{F}_{N}^{-}$-measureable random field which satisfies (1.4) and (1.5) and which agrees with $g(x, \omega)$ for $x<N$, almost surely. For example, we might let $\hat{g}_{N}$ be defined by:

$$
\hat{g}_{N}(x, \omega)= \begin{cases}g(x, \omega), & x \leq N \\ g(N, \omega), & x \geq N\end{cases}
$$

Roughly speaking, the following theorem tells us that for a time proportional to $N, z^{N}$ does not depend strongly on what lies far ahead of the interface in the region $x>N$ :

Theorem 3.4 Let $f_{0}$ be the bistable nonlinearity. Suppose that $z_{0}(x) \in C(\mathbb{R})$ satisfies

$$
0 \leq z_{0}(x) \leq 1, \quad \lim _{x \rightarrow-\infty} z_{0}(x)=1, \quad z_{0}(x) \leq K e^{-\alpha_{1} x}
$$

for some constants $K, \alpha_{1}>0$. For $N>0$, let $z^{N}(t, x, \omega)$ solve the modified initial value problem

$$
z_{t}^{N}=z_{x x}^{N}+\hat{g}_{N}(x, \omega) f_{0}\left(z^{N}\right), \quad x \in \mathbb{R}, \quad t \geq 0
$$

with initial condition $z^{N}(0, x, \omega)=z_{0}(x)$. There are non-random constants $C, C_{\tau}, r>0, p_{0} \in$ $(0,1)$, and a random variable $\tau_{N}(\omega)$ such that for almost surely with respect to $\mathbb{P}$, both $\left|\tau_{N}(\omega)\right| \leq$ $C_{\tau}$ and

$$
\sup _{x \in \mathbb{R}}\left|z^{N}(t, x, \omega)-u\left(t+\tau_{N}(\omega), x, \omega\right)\right| \leq C e^{-r t}
$$

holds for all $t \in\left[0, p_{0} N\right]$, for all $N>0$. The constants $C, C_{\tau}, r$, and $p_{0}$ are independent of $N$. 
Observe that the estimate (3.9) only applies over a time interval $t \in[0, p N]$. We should not expect the estimate to hold for all $t>0$, since eventually, the interface corresponding to $z^{N}$ will pass into the region $x>N$ where the two equations do not coincide. Let $T_{k}^{N}(\omega)$ denote the time at which the interface corresponding to $z^{N}(t, x, \omega)$ reaches the point $x=k$ :

$$
T_{k}^{N}(\omega)=\inf \left\{s \geq 0 \mid z^{N}(s, k, \omega)=\theta_{0}\right\} .
$$

The increments $\Delta T_{k}^{N}=T_{k+1}^{N}-T_{k}^{N}$ are $\mathcal{F}_{N}^{-}$-measureable random variables. Using Theorem 3.4 we derive a uniform estimate of the difference between $\Delta T_{k}^{N}$ and $\Delta T_{k}$, from which we will obtain (2.6):

Corollary 3.5 Let $f_{0}(u)$ be the bistable-type nonlinearity. There are deterministic constants $C>0, h>0$, and $s \in(0,1)$ such that

$$
\left\|\Delta T_{k}^{N}(\omega)-\Delta T_{k}\right\|_{L^{\infty}(\Omega, \mathcal{F}, \mathbb{P})} \leq C e^{-h k}
$$

holds for all $k \leq s N$, for all $N>0$. The constants $C, h$, and $s$ are independent of $N$.

As with (3.9), the estimate (3.11) holds only for $k \leq s N$, since $z^{N}$ is close to $u$ only for a time proportional to $N$ (for $t \in\left[0, p_{0} N\right]$ ). We will prove Corollary 3.5 in Section 6 .

\section{Proof of Proposition 2.2}

The bound (2.5) follows immediately from Corollary 3.2, the stationary of $\Delta \tilde{T}_{k}$, and the fact that $T_{k-j}^{z}$ is $\mathcal{F}_{0}^{+}$-measureable:

$$
\begin{aligned}
\left\|\Delta T_{k}-\mathbb{E}\left[\Delta T_{k} \mid \mathcal{F}_{j}^{+}\right]\right\|_{L^{2}(\Omega, \mathcal{F}, \mathbb{P})} & =\left\|\Delta T_{k-j}-\mathbb{E}\left[\Delta T_{k-j} \mid \mathcal{F}_{0}^{+}\right]\right\|_{L^{2}(\Omega, \mathcal{F}, \mathbb{P})} \\
& \leq\left\|\Delta T_{k-j}-\Delta T_{k-j}^{z}\right\|_{L^{2}(\Omega, \mathcal{F}, \mathbb{P})} \leq C e^{-h(k-j)}
\end{aligned}
$$

For the ignition type nonlinearity, Lemma 3.3 shows that $\Delta \tilde{T}_{k}$ is $\mathcal{F}_{j}^{-}$-measureable for any $j \geq k+1$. So, in this case, the bound (2.6) is trivial. For the bistable type nonlinearity, however, $\Delta \tilde{T}_{k}$ may not be $\mathcal{F}_{j}^{-}$-measureable for any $j \in \mathbb{Z}$. In this case, the bound (2.6) follows from Corollary 3.5. Let $s \in(0,1)$ be the constant from Corollary 3.5 , let $j-k \geq(1-s) / s$, and let $r \in\left[\frac{s(j-k)}{1-s}-1, \frac{s(j-k)}{1-s}\right]$ be a positive integer. Let $N=r+j-k$. Thus, $r \leq s N$ and $N-r=j-k$. Due to the stationary of $\Delta \tilde{T}_{k}$ and the fact that $T_{r}^{N}$ is $\mathcal{F}_{N}^{-}$-measureable, we see that

$$
\begin{aligned}
\left\|\Delta T_{k}-\mathbb{E}\left[\Delta T_{k} \mid \mathcal{F}_{j}^{-}\right]\right\|_{L^{2}(\Omega, \mathcal{F}, \mathbb{P})} & =\left\|\Delta T_{r}-\mathbb{E}\left[\Delta T_{r} \mid \mathcal{F}_{N}^{-}\right]\right\|_{L^{2}(\Omega, \mathcal{F}, \mathbb{P})} \\
& \leq\left\|\Delta T_{r}-\Delta T_{r}^{N}\right\|_{L^{2}(\Omega, \mathcal{F}, \mathbb{P})} \\
& \leq C e^{-h r} \leq C^{\prime} e^{-h s(j-k) /(1-s)} .
\end{aligned}
$$

This proves (2.6) for $j-k \geq(1-s) / s$. The general case $j \geq k$ follows from this and the fact that we always have

$$
\left\|\Delta T_{k}-\mathbb{E}\left[\Delta T_{k} \mid \mathcal{F}_{j}^{-}\right]\right\|_{L^{2}(\Omega, \mathcal{F}, \mathbb{P})} \leq \frac{1}{C_{\min }}-\frac{1}{C_{\max }}
$$

for all $j, k$, since $\left(C_{\max }\right)^{-1} \leq \Delta T_{k} \leq\left(C_{\min }\right)^{-1}$. So, by choosing the constant $C_{1}$ to be larger, if necessary, we conclude that (2.6) holds for all $j \geq k$. This proves Proposition 2.2.

\section{Proof of Theorem 3.1}

Theorem 3.1 is proved via a modification of the arguments developed in [19]. There it was shown that if $\varphi(t, x)$ solves (1.1) for $t>0$ with initial condition $\varphi(0, x)$ satisfying $(3.2)$, then $\varphi(t, x)$ converges exponentially fast to some translate of the transition front:

$$
\sup _{x \in \mathbb{R}}|\varphi(t, x)-u(t+\tau, x)| \leq C e^{-r t}
$$


for some constants $C, r>0$ and $\tau \in \mathbb{R}$ (see Theorem 1.1 of [19]). The main difference between Theorem 3.1 and the result of [19] is that $z(t, x, \omega)$ and $u(t, x, \omega)$ solve different equations. Nevertheless, the equations agree for $x>0$, which enables us to apply the strategy of [19] as the interface moves away from the origin. Here we sketch the proof and point out where modification is needed. It is easy to see that the probabilistic structure of the coefficients is superfluous in the following proof; the argument may be carried out entirely for each $\omega$ regardless of the translation invariance of $\mathbb{P}$. However, because of the application at hand, we take care to explain why the constants in (3.3) may be chosen uniformly with respect to $\mathbb{P}$.

The first step in the proof is to show that the function $z$ (which solves the modified equation (3.1)) may be trapped between two translates of the transition front $u$ (corresponding to the original equation (1.1)). Then we show that the gap between these two translates may be reduced exponentially fast as $t \rightarrow \infty$. Although the constants $C, r$, and $\tau$ depend on the initial condition $z_{0}(x)$, the rate $r$ depends only on $\alpha_{1}$, the decay rate of the initial data as $x \rightarrow \infty$. The constant $C$ depends on $\alpha_{1}$ and on the initial gap between the two translates of $u$ necessary to trap $z_{0}(x)$. Therefore, because of the uniform properties of $u$ and because the initial data $z_{0}(x)$ is deterministic, these constants may be chosen uniformly with respect to $\mathbb{P}$. In proving Theorem 3.1, we will suppose that $f_{0}(u)$ is the ignition-type nonlinearity. The case of the bistable nonlinearity (under the condition (1.6)), which is somewhat simpler, may be handled similarly with some minor modifications. Since these modifications will be highlighted later in the proof of Theorem 3.4 , we do not focus on the bistable case now.

In order to trap the solution $z$ from above and below, we construct sub- and super-solutions having the form

$$
\begin{aligned}
& \tilde{u}(t, x, \omega)=u\left(t-\zeta^{-}\left(t-t_{0}\right), x, \omega\right)-q\left(t-t_{0}\right) \Gamma\left(x-X\left(t-\zeta^{-}\left(t-t_{0}\right), \omega\right)\right) \\
& \bar{u}(t, x, \omega)=u\left(t+\zeta^{+}\left(t-t_{0}\right), x, \omega\right)+q\left(t-t_{0}\right) \Gamma\left(x-X\left(t+\zeta^{+}\left(t-t_{0}\right), \omega\right)\right) .
\end{aligned}
$$

This is a generalization of the construction developed in [6]. Here $q(t)=\epsilon(\omega) e^{-r_{0} t}$ and $\Gamma(x)$ is a smooth decreasing function satisfying $0 \leq \Gamma(x) \leq 1$ and

$$
\Gamma(x)=\Gamma_{L_{0}, \alpha}(x)=\left\{\begin{array}{cl}
1, & \text { for } x \in\left(-\infty, L_{0}-1\right] \\
e^{-\alpha\left(x-L_{0}\right)}, & \text { for } x \in\left[L_{0}+1,+\infty\right] .
\end{array}\right.
$$

The constant $L_{0}>2$ is chosen to be sufficiently large so that for almost every $\omega \in \Omega$,

$$
\begin{array}{ll}
u(t, x, \omega) \geq\left(1+\theta_{1}\right) / 2, & \forall x \leq X(t, \omega)-L_{0} / 2, \\
u(t, x, \omega) \leq \theta_{0} / 2, & \forall x \geq X(t, \omega)+L_{0} / 2,
\end{array}
$$

holds for all $t \in \mathbb{R}$, where $\theta_{1} \in\left(\theta_{0}, 1\right)$ is chosen so that $f_{0}^{\prime}(u) \leq f_{0}^{\prime}(1) / 2$ if $u \in\left[\theta_{1}, 1\right]$. That such a nonrandom constant $L_{0}$ exists follows from property (P4) of the random traveling wave. The decay rate $\alpha$ is chosen to satisfy $0<\alpha<\min \left(\lambda, \alpha_{1}\right)$. The monotonically increasing functions $\zeta^{ \pm}(t)$ are defined by

$$
\zeta^{+}(t)=\xi_{0}^{+}(\omega)+\frac{B \epsilon\left(1-e^{-r_{0} t}\right)}{r_{0}}, \quad \zeta^{-}(t)=-\xi_{0}^{-}(\omega)+\frac{B \epsilon\left(1-e^{-r_{0} t}\right)}{r_{0}}
$$

Observe that $\xi_{0}^{ \pm}(\omega)$ and $\epsilon(\omega)$ may be random variables so that the functions $\zeta^{ \pm}(t)$ and $q(t)$ may depend on $\omega \in \Omega$.

It was shown in [19] that for a suitable choice of these parameters, the functions $\bar{u}$ and $\tilde{u}$ are super- and sub- solutions, respectively, to equation (1.1). Specifically, it is necessary to choose the constant $B>0$ sufficiently large:

$$
B=\left(2 K_{f}+C_{\Gamma}\right) / \delta_{2 L_{0}},
$$

where $C_{\Gamma}=\|\Gamma\|_{C^{2}(\mathbb{R})}$ and $K_{f}$ is a uniform Lipschitz bound for $f$ :

$$
K_{f}=\left\|\sup _{x \in \mathbb{R}} \sup _{\substack{a, b \in[0,1] \\ a \neq b}} \frac{|f(x, a, \omega)-f(x, b, \omega)|}{|a-b|}\right\|_{L^{\infty}(\Omega)}<\infty .
$$


This quantity is finite, by our assumption (1.5). Then, $\epsilon(\omega)>0$ must be chosen sufficiently small:

$$
\epsilon(\omega)<\epsilon_{0}=\min \left(\theta_{0} / 4,\left(1-\theta_{1}\right) / 4,1 /(4 B)\right) .
$$

Finally, we let $\beta \in\left(0, g^{\min }\left|f_{0}^{\prime}(1)\right| / 2\right)$ and $\nu=\alpha C_{\min } / 2-\alpha^{2}$, which is positive for $0<\alpha<C_{\min } / 2$. Then $r_{0}$ must be chosen sufficiently small:

$$
r_{0} \leq \min \left(\beta, \nu, K_{f}\right) .
$$

Lemma 4.1 Let $B, K_{f}, \epsilon_{0}$, and $r_{0}$ be chosen according to (4.5) - (4.8). Suppose $\epsilon(\omega) \leq \epsilon_{0}$. Then

$$
\bar{u}_{t}-\bar{u}_{x x}-f(x, \bar{u}, \omega) \geq 0, \quad \forall t \geq t_{0}, x \in \mathbb{R} \text { such that } \bar{u}(t, x, \omega) \leq 1
$$

and

$$
\tilde{u}_{t}-\tilde{u}_{x x}-f(x, \tilde{u}, \omega) \leq 0, \quad \forall t \geq t_{0}, x \in \mathbb{R} \text { such that } \tilde{u}(t, x, \omega) \geq 0 .
$$

See Section 2.1 of [19] for details of this calculation. Although $\bar{u}$ and $\tilde{u}$ are super- and subsolutions to equation (1.1), respectively, they are not necessarily super- and sub-solutions to the modified equation (3.1), since $\hat{g}(x, \omega) \neq g(x, \omega)$ for $x \leq 0$. However, we now introduce additional conditions that will guarantee that $\tilde{u} \leq z \leq \bar{u}$ for all $x \leq 0$.

First, since $g \geq g^{\text {min }}$ and $\hat{g} \geq g^{\text {min }}$ there are deterministic constants $K_{0}>0$ and $r_{1}>0$, depending on $g^{\text {min }}$, such that

$$
\inf _{x \leq 0} u(t, x, \omega) \geq 1-K_{0} e^{-r_{1} t}, \quad \forall t \in \mathbb{R},
$$

holds with probability 1 . To see this, let $\eta(t, x)$ solve $\eta_{t}=\eta_{x x}+g^{\min } f_{0}(\eta)$ for $t>0$ with initial data

$$
\eta(0, x)=\left\{\begin{array}{cc}
\left(1+\theta_{0}\right) / 2, & x<0 \\
0, & x \geq 0
\end{array}\right.
$$

Since $u(0,0, \omega)=\theta_{0}$ almost surely, property (1.14) of $u$ implies that for $M$ sufficiently large, $\eta(0, x+M) \leq u(0, x, \omega)$ holds for all $x \in \mathbb{R}$, almost surely. Since $\eta$ is a subsolution to (1.1), the maximum principle then implies that

$$
\inf _{x \leq 0} u(t, x, \omega) \geq \inf _{x \leq 0} \eta(t, x+M)
$$

for $t \geq 0$. Furthermore, it follows from the results of [6] (e.g. Lemma 4.3, therein) that

$$
\inf _{x \leq 0} \eta(t, x+M) \geq 1-C e^{-r_{1} t}
$$

for some constants $C$ and $r_{1}$ that depend only on $g^{\text {min }}, f_{0}, M$. This implies (4.11). By the same argument, perhaps with a larger constant $K_{0}$, we also see that

$$
\inf _{x \leq 0} z(t, x, \omega) \geq 1-K_{0} e^{-r_{1} t}, \quad \forall t \in \mathbb{R},
$$

holds with probability 1 , since $\hat{g} \geq g^{\min }$ and since $\eta(0, x+M) \leq z_{0}(0, x)$ for all $x \in \mathbb{R}$, if $M$ is sufficiently large. Now using (4.11) and (4.13), we may bound $z(t, x, \omega)$ by $\bar{u}$ and $\tilde{u}$ as follows:

Lemma 4.2 Let $B, K_{f}, \epsilon_{0}$, and $r_{0}$ be chosen according to (4.5) - (4.8). Furthermore, suppose $r_{0} \in\left(0, r_{1} / 2\right)$. Then the following hold:

(i) Assume that $\epsilon(\omega) \in\left(0, \epsilon_{0}\right)$ and that for some $\xi_{0}^{-}(\omega)$ and $t_{0} \geq 0$

$$
z\left(t_{0}, x, \omega\right) \geq u\left(t_{0}+\xi_{0}^{-}, x, \omega\right)-\epsilon \Gamma\left(x-X\left(t_{0}+\xi_{0}^{-}, \omega\right)\right)
$$

holds for all $x \in \mathbb{R}$. If $\epsilon \geq K_{0} e^{-r_{0} t_{0}}$, then

$$
z(t, x, \omega) \geq u\left(t-\zeta^{-}\left(t-t_{0}\right), x, \omega\right)-\varepsilon e^{-r_{0}\left(t-t_{0}\right)} \Gamma\left(x-X\left(t-\zeta^{-}\left(t-t_{0}\right), \omega\right)\right)
$$

holds for all $t \geq t_{0}, x \in \mathbb{R}$, with $\zeta^{-}$defined by (4.4). 
(ii) Assume that $\epsilon(\omega) \in\left(0, \epsilon_{0}\right)$ and that for some $\xi_{0}^{+}(\omega)$ and $t_{0} \geq 0$

$$
z\left(t_{0}, x, \omega\right) \leq u\left(t_{0}+\xi_{0}^{+}, x, \omega\right)+\epsilon \Gamma\left(x-X\left(t_{0}+\xi_{0}^{+}, \omega\right)\right)
$$

holds for all $x \in \mathbb{R}$. If $t_{0}+\xi_{0}^{+}(\omega) \geq \max \left(0,-r_{0}^{-1} \log \left(\epsilon / 2 K_{0}\right)\right)$, then

$$
z(t, x, \omega) \leq u\left(t+\zeta^{+}\left(t-t_{0}\right), x, \omega\right)+\varepsilon e^{-r_{0}\left(t-t_{0}\right)} \Gamma\left(x-X\left(t+\zeta^{+}\left(t-t_{0}\right), \omega\right)\right)
$$

holds for all $t \geq t_{0}, x \in \mathbb{R}$, with $\zeta^{+}$is defined by (4.4).

Proof of Lemma 4.2: We first prove part (i). Suppose $\epsilon \geq K_{0} e^{-r_{0} t_{0}}$. In this case, we see that

$$
\sup _{x \leq 0} \tilde{u}(t, x, \omega) \leq \max \left(\frac{\theta_{0}}{2}, 1-\epsilon e^{-r_{0}\left(t-t_{0}\right)}\right) \leq 1-K_{0} e^{-r_{1} t} \leq \inf _{x \leq 0} z(t, x, \omega)
$$

holds for all $t \geq t_{0}$, almost surely with respect to $\mathbb{P}$. The first inequality follows from the definition of $\Gamma$ and the constant $L$. Since $\tilde{u}$ is a sub-solution to the modified equation in the region $x \geq 0$, the maximum principle then implies that $\tilde{u}(t, x, \omega) \leq z(t, x, \omega)$ holds for all $t \geq t_{0}, x \in \mathbb{R}$, as well.

Now we prove part (ii). Suppose $t_{0}+\xi_{0}^{+}(\omega) \geq \max \left(0,-r_{0}^{-1} \log \left(\epsilon / 2 K_{0}\right)\right)$ holds. Since $t+$ $\zeta^{+}\left(t-t_{0}\right) \geq t_{0}+\xi_{0}^{+} \geq 0$ for $t \geq t_{0}$, it follows that $X\left(t+\zeta^{+}\left(t-t_{0}\right)\right) \geq 0$ for $t \geq t_{0}$ and that $\Gamma\left(x-X\left(t+\zeta^{+}\left(t-t_{0}\right)\right)\right)=1$ for all $x \leq 0$ and $t \geq t_{0}$. Using this fact and (4.11), we see that

$$
\begin{aligned}
\inf _{x \leq 0} \bar{u}(t, x, \omega) & \geq \inf _{x \leq 0}\left(u\left(t+\xi_{0}^{+}, x, \omega\right)+\varepsilon e^{-r_{0}\left(t-t_{0}\right)}\right) \\
& \geq 1-K_{0} e^{-r_{1}\left(t+\xi_{0}^{+}\right)}+\varepsilon e^{-r_{0}\left(t-t_{0}\right)} \\
& \geq 1+\frac{\epsilon}{2} e^{-r_{0}\left(t-t_{0}\right)}>1=\sup _{x \in \mathbb{R}} z(t, x, \omega)
\end{aligned}
$$

holds for all $t \geq t_{0}$, almost surely with respect to $\mathbb{P}$. Therefore, since $g(x, \omega)=\hat{g}(x, \omega)$ for $x \geq 0$, the maximum principle implies that with probability $1, z(t, x, \omega) \leq \bar{u}(t, x, \omega)$ for all $x \in \mathbb{R}, t \geq t_{0}$.

Proposition 4.3 Let $r_{0}$ be chosen sufficiently small, as required by Lemma 4.2. There exist deterministic constants $T_{0}>0, T>0, r_{2}>0, K_{2}>0, \gamma \in(0,1 / 2)$ and random sequences $\left\{q_{n}(\omega)\right\}_{n \geq 0},\left\{\zeta_{n}^{+}(\omega)\right\}_{n \geq 0}$, and $\left\{\zeta_{n}^{-}(\omega)\right\}_{n \geq 0}$, such that $\mathbb{P}$-almost surely, the bounds

$$
\begin{aligned}
& z(t, x, \omega) \geq u\left(t+\zeta_{n}^{-}, x, \omega\right)-q_{n} e^{-r_{2}\left(t-t_{n}\right)} \Gamma\left(x-X\left(t+\zeta_{n}^{-}, \omega\right)\right) \\
& z(t, x, \omega) \leq u\left(t+\zeta_{n}^{+}, x, \omega\right)+q_{n} e^{-r_{2}\left(t-t_{n}\right)} \Gamma\left(x-X\left(t+\zeta_{n}^{+}, \omega\right)\right)
\end{aligned}
$$

hold for all $t \geq t_{n}=n T+T_{0}, n=0,1,2, \ldots$, and $x \in \mathbb{R}$, while the sequences $\left\{\zeta_{n}^{+}(\omega)\right\},\left\{\zeta_{n}^{-}(\omega)\right\}$, and $\left\{q_{n}(\omega)\right\}$ satisfy

$$
\begin{gathered}
0 \leq q_{n} \leq \min \left(\epsilon_{0}, \zeta_{n}^{+}-\zeta_{n}^{-}\right) \\
2^{-n} \leq \zeta_{n}^{+}-\zeta_{n}^{-} \leq K_{2}(1-\gamma / 2)^{n} \\
\zeta_{n+1}^{+} \leq \zeta_{n}^{+}+\frac{\gamma}{2 K_{2}}\left(\zeta_{n}^{+}-\zeta_{n}^{-}\right), \quad \zeta_{n+1}^{-} \geq \zeta_{n}^{-}-\frac{\gamma}{2 K_{2}}\left(\zeta_{n}^{+}-\zeta_{n}^{-}\right) \\
\zeta_{0}^{-}-1 \leq \zeta_{n}^{-} \leq \zeta_{n}^{+} \leq \zeta_{0}^{+}+1
\end{gathered}
$$

Proof of Proposition 4.3: The proposition may be proved inductively, as in [19]. For each $n$ we will apply Lemma 4.2. To make this possible, we will also require that the sequences $\left\{\zeta_{n}^{+}(\omega)\right\}$, $\left\{\zeta_{n}^{-}(\omega)\right\}$, and $\left\{q_{n}(\omega)\right\}$ satisfy

$$
\begin{gathered}
t_{n}+\zeta_{n}^{+} \geq \max \left(0,-r_{0}^{-1} \log \left(q_{n} / 2 K_{0}\right)\right) \\
q_{n} \geq K_{0} e^{-r_{0} t_{n}}
\end{gathered}
$$


for all $n \geq 0$. First, we initialize the induction argument. For $n=0$, let $t_{0}^{\prime}=\left(r_{0}\right)^{-1}\left|\log \left(\epsilon_{0} / 2 K_{0}\right)\right|$ and let $q_{0}=\left(3 \epsilon_{0}\right) / 4$. Thus, $q_{0} \geq \epsilon_{0} / 2=K_{0} e^{-r_{0} t_{0}^{\prime}}$ holds. Due to our assumptions about $z_{0}(x)$, there is a constant $\xi_{0}^{+}$sufficiently large, so that $\xi_{0}^{+}>r_{0}^{-1}\left|\log \left(q_{0} / 2 K_{0}\right)\right|$ and

$$
z_{0}(x) \leq u\left(\xi_{0}^{+}, x, \omega\right)+q_{0} \Gamma\left(x-X\left(\xi_{0}^{+}, \omega\right)\right), \forall x \in \mathbb{R}
$$

holds almost surely. Then Lemma 4.2 and the fact that $u_{t}>0$ imply

$$
\begin{aligned}
z(t, x, \omega) & \leq u\left(t+\zeta^{+}(t), x, \omega\right)+q_{0} e^{-r_{0}\left(t-t_{0}^{\prime}\right)} \Gamma\left(x-X\left(t+\zeta^{+}(t), \omega\right)\right) \\
& \leq u\left(t+\zeta_{0}^{+}, x, \omega\right)+q_{0} e^{-r_{0}\left(t-t_{0}^{\prime}\right)} \Gamma\left(x-X\left(t+\zeta_{0}^{+}, \omega\right)\right)
\end{aligned}
$$

for all $t \geq 0$ where $\zeta^{+}$is defined by (4.4) with $\epsilon=q_{0}^{\prime}$, and $\zeta_{0}^{+}:=\xi_{0}^{+}+B q_{0} / r_{0}=\sup _{t>0} \zeta^{+}(t)$.

Because $0<\alpha<\lambda$ (recall $\lambda$ in property P4), we may also choose $\xi_{0}^{-}<0$ with $\left|\xi_{0}^{-}\right|$sufficiently large so that

$$
u\left(t_{0}^{\prime}+\xi_{0}^{-}, x, \omega\right)-q_{0} \Gamma\left(x-X\left(t_{0}^{\prime}+\xi_{0}^{-}, \omega\right)\right) \leq \eta\left(t_{0}^{\prime}, x+M\right) \leq z\left(t_{0}^{\prime}, x, \omega\right)
$$

holds for all $x \in \mathbb{R}$. Since $q_{0} \geq \epsilon_{0} / 2=K_{0} e^{-r_{0} t_{0}^{\prime}}$, Lemma 4.2 also implies

$$
\begin{aligned}
z(t, x, \omega) & \geq u\left(t-\zeta^{-}\left(t-t_{0}^{\prime}\right), x, \omega\right)-q_{0} e^{-r_{0}\left(t-t_{0}^{\prime}\right)} \Gamma\left(x-X\left(t-\zeta^{-}\left(t-t_{0}^{\prime}\right), \omega\right)\right) \\
& \geq u\left(t+\zeta_{0}^{-}, x, \omega\right)-q_{0} e^{-r_{0}\left(t-t_{0}^{\prime}\right)} \Gamma\left(x-X\left(t-\zeta^{-}\left(t-t_{0}^{\prime}\right), \omega\right)\right)
\end{aligned}
$$

for all $x \in \mathbb{R}, t \geq t_{0}$. Here $\zeta^{-}(t)$ is defined by (4.4) with $\epsilon=q_{0}$, and $\zeta_{0}^{-}:=\xi_{0}^{-}-B q_{0} / r_{0}$. Now we correct the argument of $\Gamma$ in (4.29) by taking $t_{0}>t_{0}^{\prime}$, as follows. Since $\left.\mid X\left(t-\zeta^{-}\left(t-t_{0}^{\prime}\right), \omega\right)\right)-$ $\left.X\left(t+\zeta_{0}^{-}, \omega\right)\right) \mid \leq C_{\max } B q_{0} / r_{0}$, it follows from the definition of $\Gamma$ that

$$
q_{0} e^{-r_{0}\left(t-t_{0}\right)} \Gamma\left(x-X\left(t-\zeta^{-}\left(t-t_{0}^{\prime}\right), \omega\right)\right) \leq q_{0}\left(1+e^{\alpha C_{\max } B q_{0} / r_{0}}\right) e^{-r_{0}\left(t-t_{0}^{\prime}\right)} \Gamma\left(x-X\left(t+\zeta_{0}^{-}, \omega\right)\right) .
$$

Therefore, we may let $T_{0}=t_{0}=t_{0}^{\prime}+h$ with $h$ sufficiently large so that $\left(1+e^{\alpha C_{\max } B q_{0}^{\prime} / r_{0}}\right) e^{-r_{0} h}<1$. Then we have

$$
z(t, x, \omega) \geq u\left(t+\zeta_{0}^{-}, x, \omega\right)-q_{0} e^{-r_{0}\left(t-t_{0}\right)} \Gamma\left(x-X\left(t+\zeta_{0}^{-}, \omega\right)\right)
$$

This gives us the lower bound (4.19) for $n=0$. Since $t_{0}>t_{0}^{\prime}$, (4.28) implies the upper bound (4.20). Taking $\xi_{0}^{+}$larger, if necessary, we have $K_{2}:=\zeta_{0}^{+}-\zeta_{0}^{-} \geq 2^{0}$.

This establishes (4.19), (4.20), (4.21), (4.22), (4.24), (4.25), and (4.26) for $n=0$ and initializes the induction argument. Observe that $\xi_{0}^{ \pm}(\omega)=\xi_{0}^{ \pm}$may be chosen to be deterministic constants, due to the uniform properties of $u$ and the fact that $z_{0}(x)$ is deterministic. So, the initial shifts $\zeta_{0}^{+}$and $\zeta_{0}^{-}$are independent of $\omega \in \Omega$.

Next, we assume (4.19), (4.20), (4.21), (4.22), (4.24), (4.25), and (4.26) hold for some $n \geq 0$, and we complete the induction step from $n$ to $n+1$. This may be done as in the proof of Proposition 2.2 of [19] using the following strategy. We will consider three regions in the timespace domain: $\Lambda_{R}$ will denote a (random) set of points $(t, x)$ near the interface; $\Lambda_{R}^{-}$will denote a set of points behind the interface, where $u$ takes values close to $1 ; \Lambda_{R}^{+}$will note a set of points ahead of the interface where $u$ takes small values. The subscript $R>0$ controls the width of the middle region, $\Lambda_{R}$. In this region we will apply the Harnack inequality to show that $z$ must separate from the two translates of the transition front (denoted by $u_{n}^{+}$and $u_{n}^{-}$) by an amount proportional to the gap $\zeta_{n}^{+}-\zeta_{n}^{-}$. This allows us to shrink the gap while maintaining the ordering $u_{n}^{-} \leq z \leq u_{n}^{+}$within the middle region. Then we consider the outer regions $\Lambda_{R}^{+}$and $\Lambda_{R}^{-}$to show that at $t=t_{n+1}$ this ordering is attained approximately, in the sense of (4.19) and (4.20). The sets $\Lambda_{R}, \Lambda_{R}^{-}$, and $\Lambda_{R}^{+}$depend on $\omega \in \Omega$, and for $n \geq 1$ the shifts $\zeta_{n}^{ \pm}$and offsets $q_{n}$ will be random variables. However, we will suppress this dependence in our notation, and all constants chosen during the induction step will not depend on $\omega$.

In the present setting, we must modify the argument of [19] to handle the region behind the moving interface, since $u$ and $z$ do not satisfy the same equation in this region. We overcome this obstacle by using the fact that $u$ and $z$ are converging exponentially fast to 1 in the region 
$x \in(-\infty, 0]$. To apply this observation later, we now fix a constant $\tau_{0}>0$ sufficiently large so that with probability one

$$
\inf _{x \leq 0} u\left(k \tau+\zeta_{0}^{-}-1, x, \omega\right) \geq 1-K_{0} e^{-r_{1}\left(k \tau+\zeta_{0}^{-}-1\right)} \geq 1-2^{-k}
$$

holds for all $\tau \geq \tau_{0}$ and integers $k \geq 1$. This is made possible by the lower bound (4.11).

1. Near the interface. We first consider the middle region

$$
\Lambda_{R}=\left\{(t, x) \in \mathbb{R}^{2}|:| x-X\left(t+\left(\zeta_{0}^{+}+\zeta_{0}^{-}\right) / 2\right) \mid \leq R\right\}
$$

with $R>2 L_{0}>0$ chosen sufficiently large so that $\Lambda_{R}$ contains all points $(t, x)$ where both $u\left(t+\zeta_{0}^{-}-1, x\right) \leq\left(1+\theta_{1}\right) / 2$ and $u\left(t+\zeta_{0}^{+}+1, x\right) \geq \theta_{0} / 2$ hold. Due to property (P4) of $u, R$ depends only on the difference $\zeta_{0}^{+}-\zeta_{0}^{-}$. By the induction assumption, $\zeta_{0}^{-}-1 \leq \zeta_{n}^{-} \leq \zeta_{n}^{+} \leq \zeta_{0}^{+}+1$ so that either

$$
u\left(t+\zeta_{n}^{-}, x\right) \geq \frac{\left(1+\theta_{1}\right)}{2} \quad \text { or } \quad u\left(t+\zeta_{n}^{+}, x\right) \leq \frac{\theta_{0}}{2}
$$

holds for all $(t, x) \in \mathbb{R}^{2} \backslash \Lambda_{R}$, for all $n$. Observe that if $T_{0}$ is sufficiently large, then

$$
X\left(t+\left(\zeta_{0}^{+}+\zeta_{0}^{-}\right) / 2\right) \geq 3 R, \quad \forall t \geq T_{0}
$$

holds with probability one. Thus, by choosing $T_{0}$ larger, if necessary, we may guarantee that $x>0$ whenever $(t, x) \in \Lambda_{3 R}$ and $t>T_{0}$.

Let $u_{n}^{+}(t, x)$ and $u_{n}^{-}(t, x)$ denote the functions $u\left(t+\zeta_{n}^{+}, x\right)$ and $u\left(t+\zeta_{n}^{-}, x\right)$, respectively. From property (P5) of $u$, we know that for all $(t, x) \in \Lambda_{3 R}$ we have

$$
u_{n}^{+}(t, x)-u_{n}^{-}(t, x) \geq \delta_{3 R}\left(\zeta_{n}^{+}-\zeta_{n}^{-}\right)
$$

with the constant $\delta_{3 R}$ being independent of $n$ and independent of $\omega \in \Omega$. Define $\bar{s}_{n}=t_{n}+\tau_{1}$ with $\tau_{1}=\max \left(r_{2}^{-1} \log (p), \tau_{0}\right)$. Here $p>1$ is a large constant that we will set later. Then, if $\epsilon_{n}=\left(\zeta_{n}^{+}-\zeta_{n}^{-}\right) /\left(p \delta_{3 R}\right),(4.19)$ and (4.20) imply

$$
u_{n}^{+}\left(t+\epsilon_{n}, x\right) \geq u\left(t+\zeta_{n}^{+}, x\right)+\epsilon_{n} \delta_{3 R} \geq z(t, x)
$$

and

$$
u_{n}^{-}\left(t-\epsilon_{n}, x\right) \leq u\left(t+\zeta_{n}^{-}, x\right)-\epsilon_{n} \delta_{3 R} \leq z(t, x)
$$

if $t \geq \bar{s}_{n}$ and $(t, x) \in \Lambda_{3 R}$. So, the functions $u_{n}^{+}\left(t+\epsilon_{n}, x\right)-z(t, x)$ and $u_{n}^{-}\left(t-\epsilon_{n}, x\right)-z(t, x)$ are strictly positive and strictly negative, respectively, within the region $\Lambda_{3 R}$ wherever $t \geq \bar{s}_{n}$. Due to (4.34), $x>0$ whenever $(t, x) \in \Lambda_{3 R}$ and $t>t_{0}$. Therefore, the functions $u_{n}^{+}\left(t+\epsilon_{n}, x\right)$, $u_{n}^{-}\left(t-\epsilon_{n}, x\right)$, and $z$ satisfy the same equation in the region $\Lambda_{3 R}$ if $t \geq \bar{s}_{n}$. This enables us to apply the Harnack inequality to these functions.

Equation (4.35) implies that for any $t \geq \bar{s}_{n}$, either

$$
\sup _{\Lambda_{2 R} \cap\{t\} \times \mathbb{R}}\left(u_{n}^{+}\left(t+\epsilon_{n}, x\right)-z(t, x)\right) \geq \frac{\delta_{3 R}}{2}\left(\zeta_{n}^{+}-\zeta_{n}^{-}\right)
$$

or

$$
\left.\sup _{\Lambda_{2 R} \cap\{t\} \times \mathbb{R}}(z(t, x))-u_{n}^{-}\left(t-\epsilon_{n}, x\right)\right) \geq \frac{\delta_{3 R}}{2}\left(\zeta_{n}^{+}-\zeta_{n}^{-}\right)
$$

must hold. For some deterministic constants $\sigma>1$ and $\tau_{2}>1$ to be chosen later, we apply the Harnack inequality to obtain a constant $h \in(0,1)$ - depending on $\zeta_{0}^{ \pm}$and the parameter $\tau_{2}$, but independent of $\omega \in \Omega$ - such that

$$
\inf _{\Lambda_{R} \cap\left[\bar{s}_{n}+\sigma, \bar{s}_{n}+\sigma+\tau_{2}\right] \times \mathbb{R}}\left(u_{n}^{+}\left(t+\epsilon_{n}, x\right)-z(t, x)\right) \geq \frac{h \delta_{3 R}}{2}\left(\zeta_{n}^{+}-\zeta_{n}^{-}\right)
$$

if (4.36) holds at $t=\bar{s}_{n}+\sigma-1$, and

$$
\left.\inf _{\Lambda_{R} \cap\left[\bar{s}_{n}+\sigma, \bar{s}_{n}+\sigma+\tau_{2}\right] \times \mathbb{R}}(z(t, x))-u_{n}^{-}\left(t-\epsilon_{n}, x\right)\right) \geq \frac{h \delta_{3 R}}{2}\left(\zeta_{n}^{+}-\zeta_{n}^{-}\right)
$$


if (4.37) holds at $t=\bar{s}_{n}+\sigma-1$. Eventually we will choose both $\sigma$ and $\tau_{2}$ to be very large. It is important to observe here that the Harnack constant $h$ depends on $\tau_{2}$, but it does not depend on the factors $\sigma$ and $p$ or on $\bar{s}_{n}$. Assume from now on that (4.36) and (4.38) hold, the other case can be treated in a similar fashion. Let $\gamma \in\left(0, \min \left(h \delta_{3 R} / 2, \frac{1}{2}\right)\right)$. Due to property (P5) of $u$, we know that for $t \in\left[\bar{s}_{n}+\sigma, \bar{s}_{n}+\sigma+\tau_{2}\right]$ and $(t, x) \in \Lambda_{R}$ :

$$
\begin{aligned}
u_{n}^{+}\left(t-\gamma\left(\zeta_{n}^{+}-\zeta_{n}^{-}\right), x\right)-z(t, x) & \geq u_{n}^{+}\left(t+\epsilon_{n}, x\right)-z(t, x)-C\left(\epsilon_{n}+\gamma\left(\zeta_{n}^{+}-\zeta_{n}^{-}\right)\right) \\
& \geq\left(\zeta_{n}^{+}-\zeta_{n}^{-}\right)\left(h \delta_{3 R} / 2-C\left(\frac{1}{p \delta_{3 R}}+\gamma\right)\right)
\end{aligned}
$$

with $C=\left\|\sup _{t, x} u_{t}\right\|_{L^{\infty}(\Omega)}<\infty$. Since $h$ is independent of the factor $p$, we may choose $p$ large and $\gamma$ small, independently of $n$, so that the right hand side is positive for $(t, x) \in \Lambda_{R}$ and $t \in\left[\bar{s}_{n}+\sigma, \bar{s}_{n}+\sigma+\tau_{2}\right]:$

$$
u_{n}^{+}\left(t-\gamma\left(\zeta_{n}^{+}-\zeta_{n}^{-}\right), x\right)-z(t, x) \geq 0
$$

We will set $t_{n+1}=\bar{s}_{n}+\sigma+\tau_{2}$, so that $t_{n}=n T+T_{0}$ with $T=\tau_{1}+\sigma+\tau_{2}$ and $\tau_{1}, \sigma$, and $\tau_{2}$ sufficiently large.

2. Behind the interface. Next we look at the part of $\mathbb{R} \backslash \Lambda_{R}$ which is behind the interface:

$$
\Lambda_{R}^{-}:=\left\{(t, x): 0 \leq x \leq X\left(t+\left(\zeta_{0}^{+}+\zeta_{0}^{-}\right) / 2\right)-R, \quad t \in\left[\bar{s}_{n}+\sigma, \bar{s}_{n}+\sigma+\tau_{2}\right]\right\}
$$

By our choice of $R, u\left(t+\zeta_{0}^{-}-1, x\right) \geq\left(1+\theta_{1}\right) / 2$ in this region. Because $\gamma<1 / 2$, we have $\zeta_{0}^{-}-1 \leq \zeta_{n}^{-} \leq \zeta_{n}^{+}-\gamma\left(\zeta_{n}^{+}-\zeta_{n}^{-}\right)$, and thus

$$
u_{n}^{+}\left(t-\gamma\left(\zeta_{n}^{+}-\zeta_{n}^{-}\right), x\right) \geq u\left(t+\zeta_{n}^{-}, x\right) \geq u\left(t+\zeta_{0}^{-}-1, x\right) \geq\left(1+\theta_{1}\right) / 2
$$

in $\Lambda_{R}^{-}$. Moreover, for $(t, x) \in \Lambda_{R}^{-}$we have

$$
z(t, x) \geq u\left(t+\zeta_{n}^{-}, x\right)-q_{n} e^{-r_{2}\left(t-t_{n}\right)} \geq\left(1+\theta_{1}\right) / 2-\left(\zeta_{0}^{+}-\zeta_{0}^{-}\right) e^{-r_{2} \sigma} \geq \theta_{1},
$$

provided that we choose $\sigma$ sufficiently large, independently of $n$ and $\omega \in \Omega$. Hence, the function

$$
v(t, x):=u_{n}^{+}\left(t-\gamma\left(\zeta_{n}^{+}-\zeta_{n}^{-}\right), x\right)-z(t, x)
$$

satisfies

$$
v_{t}-v_{x x}=f\left(u\left(t+\zeta_{n}^{+}-\gamma\left(\zeta_{n}^{+}-\zeta_{n}^{-}\right), x\right)-f(z(t, x))=a(t, x) v,\right.
$$

with $a(t, x) \leq-\beta<0$ in $\Lambda_{R}^{-}$.

Now we use the comparison principle to bound $v$ from below at time $t_{n}=\bar{s}_{n}+\sigma+\tau_{2}$. Because $u_{t}>0$, the right boundary of $\Lambda_{R}^{-}$, which is the set

$$
\left\{\left(t, X\left(t+\left(\zeta_{0}^{+}+\zeta_{0}^{-}\right) / 2\right)-R\right) \mid t \in\left[\bar{s}_{n}+\sigma, \bar{s}_{n}+\sigma+\tau_{2}\right]\right\}
$$

is a smooth curve, and $v(t, x) \geq 0$ on this curve because of (4.40). Initially, at time $t=\bar{s}_{n}+\sigma$ we have, with $C=\left\|\sup _{t, x} u_{t}\right\|_{L^{\infty}(\Omega)}$ :

$$
\begin{aligned}
v\left(\bar{s}_{n}+\sigma, x\right) & =u\left(\bar{s}_{n}+\sigma+\zeta_{n}^{+}-\gamma\left(\zeta_{n}^{+}-\zeta_{n}^{-}\right), x\right)-z\left(\bar{s}_{n}+\sigma, x\right) \\
& \geq-q_{n} e^{-r_{2}\left(\tau_{1}+\sigma\right)}-C \gamma\left(\zeta_{n}^{+}-\zeta_{n}^{-}\right) \geq-\left(\zeta_{n}^{+}-\zeta_{n}^{-}\right) e^{-r_{2} \sigma}-C \gamma\left(\zeta_{n}^{+}-\zeta_{n}^{-}\right)
\end{aligned}
$$

for all $x \in \mathbb{R}$. We used here the induction assumption $0 \leq q_{n} \leq \zeta_{n}^{+}-\zeta_{n}^{-}$. The lower bound (4.31) implies that at the left boundary where $x=0$ we have

$$
\begin{aligned}
v(t, 0, \omega) & \geq u\left(t+\zeta_{n}^{+}-\gamma\left(\zeta_{n}^{+}-\zeta_{n}^{-}\right), 0, \omega\right)-1 \\
& \geq u\left(t+\zeta_{0}^{-}-1,0, \omega\right)-1 \\
& \geq-2^{-(k+n+1)}
\end{aligned}
$$


for all $t \geq k \tau_{0}+\bar{s}_{n} \geq(k+n+1) \tau_{0}$. Therefore, if $\lambda=\min \left(\left(\tau_{0}\right)^{-1} \log 2, \beta\right)>0$, then

$$
v(t, 0, \omega) \geq-2^{-n} e^{-\lambda\left(t-\bar{s}_{n}\right)}
$$

for all $t \geq \bar{s}_{n}+\sigma$. Consequently, applying the comparison principle to (4.41) in the temporalspatial domain $\Lambda_{R}^{-}$we conclude that

$$
v(t, x) \geq-2^{-n} e^{-\lambda \sigma} e^{-\lambda\left(t-\bar{s}_{n}-\sigma\right)}-e^{-\beta\left(t-\bar{s}_{n}-\sigma\right)} C\left[e^{-r_{2} \sigma}+\gamma\right]\left(\zeta_{n}^{+}-\zeta_{n}^{-}\right) \quad \text { for }(t, x) \in \Lambda_{R}^{-} .
$$

By the induction assumption $\zeta_{n}^{+}-\zeta_{n}^{-} \geq 2^{-n}$, so

$$
\begin{aligned}
v(t, x) & \geq-\left(\zeta_{n}^{+}-\zeta_{n}^{-}\right) e^{-\lambda \sigma} e^{-\lambda\left(t-\bar{s}_{n}-\sigma\right)}-e^{-\beta\left(t-\bar{s}_{n}-\sigma\right)} C\left[e^{-r_{2} \sigma}+\gamma\right]\left(\zeta_{n}^{+}-\zeta_{n}^{-}\right) \\
& \geq-e^{-\lambda\left(t-\bar{s}_{n}-\sigma\right)} C\left[2 e^{-r_{2} \sigma}+\gamma\right]\left(\zeta_{n}^{+}-\zeta_{n}^{-}\right) \quad \text { for }(t, x) \in \Lambda_{R}^{-} .
\end{aligned}
$$

3. Ahead of the interface. Finally we consider the region in $\mathbb{R} \backslash \Lambda_{R}$ ahead of interface:

$$
\Lambda_{R}^{+}:=\left\{(t, x): x \geq X\left(t+\left(\zeta_{0}^{+}+\zeta_{0}^{-}\right) / 2\right)+R, \quad t \in\left[\bar{s}_{n}+\sigma, \bar{s}_{n}+\sigma+\tau_{2}\right]\right\} .
$$

By our choice of $R, u\left(t+\zeta_{0}^{+}+1, x\right) \leq \theta_{0} / 2$ in this region. In a manner identical to the argument in [19] we may show that

$$
v(t, x) \geq-C\left(\zeta_{n}^{+}-\zeta_{n}^{-}\right)\left(e^{-r_{2} \sigma}+\gamma\right) e^{-\alpha\left(x-X\left(t+\zeta_{n}^{+}\right)\right)-\nu\left(t-\bar{s}_{n}-\sigma\right)} \text { for }(t, x) \in \Lambda_{R}^{+}
$$

where $C=\left\|\sup _{t, x} u_{t}\right\|_{L^{\infty}(\Omega)}<\infty$.

4. Shrinking the gap $\zeta_{n}^{+}-\zeta_{n}^{-}$. Now we combine the estimates (4.40), (4.45) and (4.46). We have shown that if (4.36) holds (rather than (4.37)) then at times $t \in\left(\bar{s}_{n}+\sigma, \bar{s}_{n}+\sigma+\tau_{2}\right)$ we have

$$
z(t, x) \leq u\left(t+\zeta_{n}^{+}-\gamma\left(\zeta_{n}^{+}-\zeta_{n}^{-}\right), x\right)
$$

for $(t, x) \in \Lambda_{R}$,

$$
z(t, x) \leq u\left(t+\zeta_{n}^{+}-\gamma\left(\zeta_{n}^{+}-\zeta_{n}^{-}\right), x\right)+C\left(2 e^{-r_{2} \sigma}+\gamma\right)\left(\zeta_{n}^{+}-\zeta_{n}^{-}\right) e^{-\lambda\left(t-\bar{s}_{n}-\sigma\right)}
$$

for $(t, x) \in \Lambda_{R}^{-}$, and

$$
z(t, x) \leq u\left(t+\zeta_{n}^{+}-\gamma\left(\zeta_{n}^{+}-\zeta_{n}^{-}\right), x\right)+C\left(e^{-r_{2} \sigma}+\gamma\right)\left(\zeta_{n}^{+}-\zeta_{n}^{-}\right) e^{-\alpha\left(x-X\left(t+\zeta_{n}^{+}\right)\right)-\nu\left(t-\bar{s}_{n}-\sigma\right)}
$$

for $(t, x) \in \Lambda_{R}^{+}$. We also still have the lower bound:

$$
z(t, x) \geq u\left(t+\zeta_{n}^{-}, x\right)-q_{n} e^{-r_{2}\left(t-t_{n}\right)} \Gamma\left(x-X\left(t+\zeta_{n}^{-}\right)\right)
$$

for all $t \geq t_{n}$ and $x \in \mathbb{R}$, and by the induction assumption $q_{n} \leq\left(\zeta_{n}^{+}-\zeta_{n}^{-}\right)$.

We may now set $t_{n+1}=\bar{s}_{n}+\sigma+\tau_{2}$, so that $t_{n}=n T+T_{0}$ with $T=\tau_{1}+\sigma+\tau_{2}$. We define the new shifts as

$$
\zeta_{n+1 / 2}^{+}=\zeta_{n}^{+}-\gamma\left(\zeta_{n}^{+}-\zeta_{n}^{-}\right), \quad \zeta_{n+1 / 2}^{-}=\zeta_{n}^{-},
$$

and the new correction $q_{n+1 / 2}=C\left(\zeta_{n}^{+}-\zeta_{n}^{-}\right)\left(e^{-r_{2} \sigma}+\gamma\right) e^{-r_{2} \tau_{2}}$. Here we assume $r_{2}$ is chosen to be $r_{2}<\min (\nu, \lambda) \leq \beta$. Then for $t=t_{n+1}$ we conclude that

$$
\begin{aligned}
& z\left(t_{n+1}, x\right) \geq u\left(t_{n+1}+\zeta_{n+1 / 2}^{-}, x\right)-q_{n+1 / 2} \Gamma\left(x-X\left(t_{n+1}+\zeta_{n+1 / 2}^{-}\right)\right) \\
& z\left(t_{n+1}, x\right) \leq u\left(t_{n+1}+\zeta_{n+1 / 2}^{+}, x\right)+q_{n+1 / 2} \Gamma\left(x-X\left(t_{n+1}+\zeta_{n}^{+}\right)\right) .
\end{aligned}
$$

Just as in [19] we may correct the argument of the function $\Gamma$ in (4.51) to obtain

$$
z\left(t_{n+1}, x\right) \leq u\left(t_{n+1}+\zeta_{n+1 / 2}^{+}, x\right)+q_{n+1 / 2}^{\prime} \Gamma\left(x-X\left(t_{n+1}+\zeta_{n+1 / 2}^{+}\right)\right) .
$$


Here we have defined $q_{n+1 / 2}^{\prime}=C q_{n+1 / 2}$, with $C>1+e^{\alpha C_{\max } B \epsilon_{0} / r}$ a large non-random constant depending only on $C_{\max }$ and $\zeta_{0}^{+}-\zeta_{0}^{-}$.

We will conclude the induction step by applying Lemma 4.2 to (4.50) and (4.52). To this end, we set

$$
\begin{aligned}
& q_{n+1}=q_{n+1 / 2}^{\prime}=\left(\zeta_{n}^{+}-\zeta_{n}^{-}\right) C\left(e^{-r_{2} \sigma}+\gamma\right) e^{-r_{2} \tau_{2}} \\
& \zeta_{n+1}^{+}=\zeta_{n+1 / 2}^{+}+K_{3} q_{n+1 / 2}^{\prime}=\zeta_{n}^{+}-\gamma\left(\zeta_{n}^{+}-\zeta_{n}^{-}\right)+K_{3}\left(\zeta_{n}^{+}-\zeta_{n}^{-}\right) C\left(e^{-r_{2} \sigma}+\gamma\right) e^{-r_{2} \tau_{2}} \\
& \zeta_{n+1}^{-}=\zeta_{n+1 / 2}^{-}-K_{3} q_{n+1 / 2}^{\prime}=\zeta_{n}^{-}-C K_{3}\left(\zeta_{n}^{+}-\zeta_{n}^{-}\right)\left(e^{-r_{2} \sigma}+\gamma\right) e^{-r_{2} \tau_{2}}
\end{aligned}
$$

and $K_{3}=B / r_{0}$. The constant $K_{3}$ comes from the observation that $\left|\zeta^{ \pm}(t)-\zeta^{ \pm}(0)\right| \leq K_{3} \epsilon$. Let us verify (4.21)-(4.26). First, from (4.53) we see that

$$
q_{n+1}=\left(\zeta_{n}^{+}-\zeta_{n}^{-}\right) C\left(e^{-r_{2} \sigma}+\gamma\right) e^{-r_{2} \tau_{2}} \leq \min \left(\epsilon_{0}, \zeta_{n+1}^{+}-\zeta_{n+1}^{-}\right),
$$

provided that $\tau_{2}$ is sufficiently large. So, (4.21) holds for $n+1$. Next,

$$
\zeta_{n+1}^{+}-\zeta_{n+1}^{-}=\left(\zeta_{n}^{+}-\zeta_{n}^{-}\right)\left(1-\gamma+2 K_{3} C\left(e^{-r_{2} \sigma}+\gamma\right) e^{-r_{2} \tau_{2}}\right) .
$$

The parameter $\gamma \in(0,1 / 2)$ depends on the Harnack constant over the time interval of length $\tau_{2}$. By first taking $\tau_{2}$ sufficiently large and then choosing $\sigma$ large enough, independently of $\omega \in \Omega$, we can ensure that

$$
(1-\gamma)\left(\zeta_{n}^{+}-\zeta_{n}^{-}\right) \leq \zeta_{n+1}^{+}-\zeta_{n+1}^{-} \leq(1-\gamma / 2)\left(\zeta_{n}^{+}-\zeta_{n}^{-}\right)
$$

Therefore, by the induction assumption $2^{-(n+1)} \leq \zeta_{n+1}^{+}-\zeta_{n+1}^{-} \leq K_{2}(1-\gamma / 2)^{n+1}$ holds, where $K_{2}=\left(\zeta_{0}^{+}-\zeta_{0}^{-}\right)$. Thus (4.22) holds for $n+1$.

The new values in (4.53) were obtained under the assumption that (4.36) rather than (4.37) holds, yet the geometric bound (4.55) holds in either case. In either case we also find that

$$
\zeta_{n}^{-}-\frac{\gamma}{2 K_{2}}\left(\zeta_{n}^{+}-\zeta_{n}^{-}\right) \leq \zeta_{n+1}^{-} \leq \zeta_{n+1}^{+} \leq \zeta_{n}^{+}+\frac{\gamma}{2 K_{2}}\left(\zeta_{n}^{+}-\zeta_{n}^{-}\right)
$$

provided that $\tau_{2}$ and $\sigma$ are sufficiently large. This is (4.23). Now we verify (4.24). By the induction assumption and what we have shown already, $\zeta_{n+1}^{+} \leq \zeta_{n}^{+}+\left(\gamma / 2 K_{2}\right)\left(\zeta_{n}^{+}-\zeta_{n}^{-}\right) \leq$ $\zeta_{n}^{+}+(\gamma / 2)(1-\gamma / 2)^{n}$. Therefore, $\zeta_{n+1}^{+} \leq \zeta_{0}^{+}+(\gamma / 2) \sum_{k=0}^{n}(1-\gamma / 2)^{k} \leq \zeta_{0}^{+}+1$. So, (4.24) holds for $\zeta_{n+1}^{+}$.

Since $q_{n+1} \geq\left(\zeta_{n}^{+}-\zeta_{n}^{-}\right) C \gamma e^{-r_{2} \tau_{2}} \geq 2^{-n} \gamma e^{-r_{2} \tau_{2}}$, we may increase $\sigma$ or $\tau_{1}$ as necessary to obtain $q_{n+1} \geq K_{0} e^{-r_{0} t_{n+1}}$. Thus, (4.26) holds for $n+1$. Finally,

$$
\begin{aligned}
t_{n+1}+\zeta_{n+1}^{+} & \geq t_{n}+\zeta_{n}^{+}+T-\gamma\left(\zeta_{n}^{+}-\zeta_{n}^{-}\right) \\
& \geq r_{0}^{-1}\left|\log \left(\frac{q_{n}}{2 K_{0}}\right)\right|+T-\gamma K_{2}(1-\gamma / 2)^{n} \\
& \geq r_{0}^{-1}\left|\log \left(\frac{q_{n+1}}{2 K_{0}}\right)\right|-r_{0}^{-1}\left|\log \left(\frac{q_{n+1}}{q_{n}}\right)\right|+T-\gamma K_{2}(1-\gamma / 2)^{n}
\end{aligned}
$$

From (4.55) and the definition of $q_{n}$, the ratio $q_{n+1} / q_{n}$ satisfies $(1-\gamma) \leq q_{n+1} / q_{n} \leq(1-\gamma / 2)$. Therefore, since $T=\tau_{1}+\sigma+\tau_{2}$ where $\gamma$ is independent of $\tau_{1}$ and $\sigma$, we may increase $\sigma$ or $\tau_{1}$ as necessary to obtain $t_{n+1}+\zeta_{n+1}^{+} \geq r_{0}^{-1}\left|\log \left(\frac{q_{n+1}}{2 K_{0}}\right)\right|$ which shows that (4.25) holds for $t_{n+1}$.

Since (4.25) holds for $n+1$, Lemma 4.2 applied to (4.52) implies that for $t \geq t_{n+1}$

$$
z(t, x) \leq u\left(t+\zeta_{n+1}^{+}, x\right)+q_{n+1} e^{-r_{2}\left(t-t_{n+1}\right)} \Gamma\left(x-X\left(t+\zeta_{n+1}^{+}\right)\right)
$$

holds for all $x$. Here we have used the fact that $u_{t}>0$, as we did at (4.28). Since (4.26) also holds for $n+1$, Lemma 4.2 applied to (4.50) implies that for $t \geq t_{n+1}$ we have

$$
z(t, x) \geq u\left(t+\zeta_{n+1}^{-}, x\right)-\left(q_{n+1 / 2}\right) e^{-r_{2}\left(t-t_{n+1}\right)} \Gamma\left(x-X\left(t-v\left(t-t_{n+1}\right)\right)\right)
$$


where $v(s)=-\zeta_{n+1}^{-}+\left(r_{0}^{-1} B q_{n+1 / 2}^{\prime}\right)\left(1-e^{-r_{0} s}\right)$. Therefore, since $\left|X\left(t-v\left(t-t_{n+1}\right)\right)-X\left(t+\zeta_{n+1}^{-}\right)\right| \leq$ $C_{\text {max }} B \epsilon_{0} / r_{0}$, we conclude that

$$
\left.z(t, x) \geq u\left(t+\zeta_{n+1}^{-}, x\right)-q_{n+1} e^{-r_{2}\left(t-t_{n+1}\right)} \Gamma\left(x-X\left(t+\zeta_{n+1}^{-}\right)\right)\right)
$$

holds for all $x \in \mathbb{R}$. (Recall $q_{n+1}=C q_{n+1 / 2}$, with $C>1+e^{\alpha C_{\max } B \epsilon_{0} / r}$.)

This completes the proof of Proposition 4.3.

Theorem 3.1 now follows directly from Proposition 4.3. From (4.23) - (4.24) it is easy to see that for almost every $\omega \in \Omega,\left\{\zeta_{n}^{+}(\omega)\right\}_{n}$ is a Cauchy sequence and that there is a random variable $\tau(\omega)$ and a constant $C$ such that

$$
\left|\zeta_{n}^{+}(\omega)-\tau(\omega)\right| \leq C\left(1-\frac{\gamma}{2}\right)^{n}, \quad \forall n \geq 0
$$

In particular, $|\tau(\omega)|$ is uniformly bounded since $\left|\zeta_{n}^{+}\right|$is uniformly bounded. Since $\left|q_{n}(\omega)\right| \leq$ $C\left(1-\frac{\gamma}{2}\right)^{n}$ also holds, this implies (3.3). This completes the proof of Theorem 3.1.

\section{Proof of Theorem 3.4.}

Now $f_{0}(u)$ is the bistable type nonlinearity. We follow the same strategy as in the proof of Theorem 3.1 using sub- and super-solutions. Again, the argument is similar to that in [19]; the main new difficulty is the fact that $z^{N}$ and $u$ satisfy different equations, which agree only for $x \leq N$. In order to trap the solution $z$ from above and below, we construct super- and sub-solutions having the form

$$
\begin{aligned}
& \bar{u}(t, x, \omega)=u\left(t+\zeta^{+}\left(t-t_{0}\right), x, \omega\right)+q\left(t-t_{0}\right) \\
& \tilde{u}(t, x, \omega)=u\left(t-\zeta^{-}\left(t-t_{0}\right), x, \omega\right)-q\left(t-t_{0}\right) .
\end{aligned}
$$

This is a variation of the construction first developed in [6]. Here $q(t)=\epsilon(\omega) e^{-r_{0} t}$. The monotonically increasing functions $\zeta^{ \pm}(t)$ are defined by

$$
\zeta^{+}(t)=\xi_{0}^{+}(\omega)+\frac{B \epsilon\left(1-e^{-r_{0} t}\right)}{r_{0}}, \quad \zeta^{-}(t)=-\xi_{0}^{-}(\omega)+\frac{B \epsilon\left(1-e^{-r_{0} t}\right)}{r_{0}}
$$

Observe that $\xi_{0}^{ \pm}(\omega)$ and $\epsilon(\omega)$ may be random variables so that the functions $\zeta^{ \pm}(t)$ and $q(t)$ may depend on $\omega \in \Omega$.

For a suitable choice of these parameters, the functions $\bar{u}$ and $\tilde{u}$ are super- and sub-solutions, respectively, to equation (1.1). We choose a constant $L_{0}>2$ sufficiently large so that for almost every $\omega \in \Omega$,

$$
\begin{array}{ll}
u(t, x, \omega) \geq\left(1+\theta_{1}\right) / 2, & \forall x \leq X(t, \omega)-L_{0} / 2, \\
u(t, x, \omega) \leq \theta_{2} / 2, & \forall x \geq X(t, \omega)+L_{0} / 2,
\end{array}
$$

holds for all $t \in \mathbb{R}$, where $\theta_{1} \in\left(\theta_{0}, 1\right)$ is chosen so that $f_{0}^{\prime}(u) \leq f_{0}^{\prime}(1) / 2<0$ if $u \in\left[\theta_{1}, 1\right]$, and $\theta_{2} \in\left(0, \theta_{0}\right)$ is chosen so that $f_{0}^{\prime}(u) \leq f_{0}^{\prime}(0) / 2<0$ if $u \in\left[0, \theta_{2}\right]$. It is necessary to choose the constant $B>0$ sufficiently large, according to (4.5). Then, $\epsilon(\omega)>0$ must be chosen sufficiently small:

$$
\epsilon(\omega)<\epsilon_{0}=\min \left(\theta_{2} / 4,\left(1-\theta_{1}\right) / 4,1 /(4 B)\right) .
$$

Finally, we let $\beta \in\left(0, \frac{g^{\text {min }}}{2} \min \left(\left|f_{0}^{\prime}(0)\right|,\left|f^{\prime}(1)\right|\right)\right)$ Then $r_{0}$ must be chosen sufficiently small:

$$
r_{0} \leq \min \left(\beta, K_{f}\right),
$$

where the constant $K_{f}$ is defined by (4.6). 
Lemma 5.1 Let $B, \epsilon_{0}$, and $r_{0}$ be chosen according to (4.5), (5.3), and (5.4). Suppose $\epsilon(\omega) \leq \epsilon_{0}$. Then

$$
\bar{u}_{t}-\bar{u}_{x x}-f(x, \bar{u}, \omega) \geq 0, \quad \forall t \geq t_{0}, x \in \mathbb{R} \text { such that } \bar{u}(t, x, \omega) \leq 1
$$

and

$$
\tilde{u}_{t}-\tilde{u}_{x x}-f(x, \tilde{u}, \omega) \leq 0, \quad \forall t \geq t_{0}, x \in \mathbb{R} \text { such that } \tilde{u}(t, x, \omega) \geq 0 .
$$

This computation is almost identical to the one carried out in [19], so we omit the proof. Using the sub- and super-solutions constructed in this way, we may bound the solution $z(t, x, \omega)$ as follows:

Lemma 5.2 Let $B, \epsilon_{0}$ be chosen according to (4.5), (5.3). If $r_{0}$ is sufficiently small, there are positive constants $p_{0}, p_{1}, p_{2}$, and $p_{3}$ such that the following hold:

(i) Assume that $\epsilon(\omega) \in\left(0, \epsilon_{0}\right)$ and that for some $\xi_{0}^{-}(\omega)$ and $t_{0} \in\left[0, p_{0} N\right)$

$$
z^{N}\left(t_{0}, x, \omega\right) \geq u\left(t_{0}+\xi_{0}^{-}, x, \omega\right)-\epsilon
$$

holds for all $x \in(-\infty, N]$. If $\xi_{0}^{-} \leq p_{3} \log (\epsilon)-p_{1}+p_{2} N$, then

$$
z^{N}(t, x, \omega) \geq u\left(t-\zeta^{-}\left(t-t_{0}\right), x, \omega\right)-\varepsilon e^{-r_{0}\left(t-t_{0}\right)}
$$

holds for all $t \in\left[t_{0}, p_{0} N\right], x \in \mathbb{R}$, with $\zeta^{-}$defined by (5.2). The constants $p_{0}, p_{1}, p_{2}$, and $p_{3}$ are deterministic.

(ii) Assume that $\epsilon(\omega) \in\left(0, \epsilon_{0}\right)$ and that for some $\xi_{0}^{+}(\omega)$ and $t_{0} \in\left[0, p_{0} N\right)$

$$
z^{N}\left(t_{0}, x, \omega\right) \leq u\left(t_{0}+\xi_{0}^{+}, x, \omega\right)+\epsilon
$$

holds for all $x \in(-\infty, N]$. If $0 \leq \log (\epsilon)-p_{1}+p_{2} N$, then

$$
z^{N}(t, x, \omega) \leq u\left(t+\zeta^{+}\left(t-t_{0}\right), x, \omega\right)+\varepsilon e^{-r_{0}\left(t-t_{0}\right)}
$$

holds for all $t \in\left[t_{0}, p_{0} N\right], x \in(-\infty, N]$, with $\zeta^{+}$is defined by (5.2).

Proof of Lemma 5.2: We first prove part (i). Suppose $r_{0}$ is sufficiently small so that (5.4) is satisfied. Let $\tilde{u}$ be defined by (5.1). By Lemma 5.1, the function $\tilde{u}$ is a sub-solution to equation (1.1), so it is a sub-solution to the modified equation (3.8) in the region $x \in(-\infty, N]$. We now show that $\tilde{u}(t, x, \omega) \leq 0$ for $x \geq N$ and $t \in\left[t_{0}, p_{0} N\right]$.

Due to our assumption (1.6) there are deterministic constants $C$ and $r_{1}$, depending on $g^{\text {min }}$, $g^{\max }$, and $f_{0}$ such that

$$
u(t, x, \omega) \leq C e^{-r_{1}(x-X(t, \omega))}, \quad \forall t \in \mathbb{R},
$$

holds with probability 1 . Taking $r_{0}$ smaller, if necessary, we may assume $r_{0} \in\left(0, r_{1} / 2\right)$.

$$
\begin{aligned}
\sup _{x \geq N+X\left(t_{0}, \omega\right)} \tilde{u}(t, x, \omega) & \leq \sup _{x \geq N+X\left(t_{0}, \omega\right)}\left(u\left(t+\xi_{0}^{-}, x, \omega\right)-\varepsilon e^{-r_{0}\left(t-t_{0}\right)}\right) \\
& \leq \sup _{x \geq N+X\left(t_{0}, \omega\right)} C e^{-r_{1}\left(x-X\left(t+\xi_{0}^{-}, \omega\right)\right)}-\varepsilon e^{-r_{0}\left(t-t_{0}\right)} \\
& =\sup _{x \geq N+X\left(t_{0}, \omega\right)} C e^{-r_{1}\left(x-X\left(t_{0}, \omega\right)+X\left(t_{0}, \omega\right)-X\left(t+\xi_{0}^{-}, \omega\right)\right)}-\varepsilon e^{-r_{0}\left(t-t_{0}\right)} \\
& \leq C e^{-r_{1} N} e^{r_{1} C_{\max }\left(t+\xi_{0}^{-}-t_{0}\right)}-\varepsilon e^{-r_{0}\left(t-t_{0}\right)}
\end{aligned}
$$

If $t \in\left[t_{0}, p_{0} N\right]$, then $\left(t-t_{0}\right) \leq p_{0} N$, and this last term is negative if

$$
\xi_{0}^{-} \leq \frac{1}{C_{\max } r_{1}} \log \left(\varepsilon / 2 C_{1}\right)+N\left(\frac{1}{C_{\max }}-\frac{r_{0} p_{0}}{C_{\max } r_{1}}-p_{0}\right) .
$$

Let $p_{0} \leq 1 /\left(4\left(1+C_{\max }\right)\right)$. Then it suffices for

$$
\xi_{0}^{-} \leq \frac{1}{C_{\max } r_{1}} \log \left(\varepsilon / 2 C_{1}\right)+\frac{N}{2 C_{\max }}=p_{3} \log (\epsilon)-p_{1}+p_{2} N,
$$


with $p_{3}=\left(C_{\max } r_{1}\right)^{-1}$. This implies that $\tilde{u} \leq z^{N}$ for all $x \geq N$, while for $x \leq N$, $\tilde{u}$ is a sub-solution to equation (1.1). Therefore, the comparison principle implies

$$
z^{N}(t, x, \omega) \geq \tilde{u}(t, x, \omega), \quad \forall x \in \mathbb{R}, \quad t \in\left[t_{0}, p_{0} N\right]
$$

holds with probability 1.

Now we prove part (ii). Let $\bar{u}$ be defined by (5.1). By Lemma 5.1, $\bar{u}$ is a super-solution to equation (1.1), but it is not globally a super-solution to the modified equation (3.8) since $\hat{g}_{N}$ may not agree with $g$ for $x>N$. However, we will show that

$$
\bar{u}(t, N, \omega) \geq z^{N}(t, N, \omega), \quad \forall t \in\left[t_{0}, p_{0} N\right] .
$$

The hypotheses on $z_{0}$ imply that

$$
z^{N}(t, x, \omega) \leq K_{4} e^{-r_{3}\left(x-C_{\max } t\right)} .
$$

for some deterministic constant $r_{3}>0$. Therefore (5.12) holds if

$$
u\left(t+\zeta^{+}\left(t-t_{0}\right), N, \omega\right)+\epsilon e^{-r_{0}\left(t-t_{0}\right)} \geq K_{4} e^{-r_{3}\left(N-C_{\max } t\right)}
$$

holds for $t \in\left[t_{0}, p_{0} N\right]$. Since $u>0$, this holds under the condition

$$
\log (\epsilon) \geq \log \left(K_{4}\right)+\left(\left(r_{0}+r_{3} C_{\max }\right) p_{0}-r_{3}\right) N .
$$

Now by choosing $p_{0}$ smaller, $p_{1}$ larger, and $p_{2}$ smaller, if necessary, we see that (5.12) holds under the condition $\log (\epsilon) \geq p_{1}-p_{2} N$. The hypothesis (5.9) implies $\bar{u}\left(t_{0}, x, \omega\right) \geq z^{N}\left(t_{0}, x, \omega\right)$ for all $x \in \mathbb{R}, \mathbb{P}$-almost surely. Therefore, (5.12) and the comparison principle implies that $\bar{u} \geq z^{N}$ for all $x \in(-\infty, N]$ and all $t \in\left[t_{0}, p_{0} N\right]$. This proves (ii).

Proposition 5.3 Let $\epsilon_{0}$ be defined by (5.3). Let $r_{0}$ be chosen sufficiently small, as required by Lemma 5.2. There exist deterministic constants $T>1, r_{2}>0, K_{1}>0, \gamma \in(0,1 / 2)$, and $\ell \in\left(0, p_{0} / T\right)$ such that for all $N$ sufficiently large, there are random sequences $\left\{q_{n}(\omega)\right\}_{n \geq 0}$, $\left\{\zeta_{n}^{+}(\omega)\right\}_{n \geq 0}$, and $\left\{\zeta_{n}^{-}(\omega)\right\}_{n \geq 0}$, such that $\mathbb{P}$-almost surely the following hold:

(i) For all integers $n \in[0, \ell N-1]$,

$$
\begin{gathered}
z^{N}(t, x, \omega) \geq u\left(t+\zeta_{n}^{-}, x, \omega\right)-q_{n} e^{-r_{2}\left(t-t_{n}\right)} \\
z^{N}(t, x, \omega) \leq u\left(t+\zeta_{n}^{+}, x, \omega\right)+q_{n} e^{-r_{2}\left(t-t_{n}\right)},
\end{gathered}
$$

holds for all $t \in\left[t_{n}, p_{0} N\right]$ and $x \in(-\infty, N]$, where $t_{n}=n T$.

(ii) For all integers $n \in[0, \ell N-1],\left\{\zeta_{n}^{+}(\omega)\right\},\left\{\zeta_{n}^{-}(\omega)\right\}$, and $\left\{q_{n}(\omega)\right\}$ satisfy

$$
\begin{gathered}
0 \leq q_{n} \leq \min \left(\epsilon_{0}, \zeta_{n}^{+}-\zeta_{n}^{-}\right) \\
2^{-n} \leq \zeta_{n}^{+}-\zeta_{n}^{-} \leq K_{1}(1-\gamma / 2)^{n} \\
\zeta_{n+1}^{+} \leq \zeta_{n}^{+}+\frac{\gamma}{2 K_{1}}\left(\zeta_{n}^{+}-\zeta_{n}^{-}\right), \quad \zeta_{n+1}^{-} \geq \zeta_{n}^{-}-\frac{\gamma}{2 K_{1}}\left(\zeta_{n}^{+}-\zeta_{n}^{-}\right) . \\
\zeta_{0}^{-}-1 \leq \zeta_{n}^{-} \leq \zeta_{n}^{+} \leq \zeta_{0}^{+}+1 \\
\max \left(\zeta_{n}^{-}, 0\right) \leq p_{3} \log \left(q_{n}\right)-p_{1}+p_{2} N
\end{gathered}
$$

Proof of Proposition 5.3: This proposition is analogous to Proposition 4.3, and the structure of the proof is the same. First, for $n=0$, let $t_{0}=0$ and $q_{0}=\epsilon_{0}<1$. If $N$ is sufficiently large, then $0<p_{3} \log \left(q_{0}\right)-p_{1}+p_{2} N$. There are constants $\xi_{0}^{+}$and $\left|\xi_{0}^{-}\right|$sufficiently large, so that both $\xi_{0}^{-} \leq 0<p_{3} \log \left(q_{0}\right)-p_{1}+p_{2} N$ and

$$
u\left(\xi_{0}^{-}, x, \omega\right)-q_{0} \leq z_{0}(x) \leq u\left(\xi_{0}^{+}, x, \omega\right)+q_{0}, \forall x \in \mathbb{R}
$$

hold almost surely. Then Lemma 5.2 implies that

$$
u\left(t+\zeta_{0}^{-}, x, \omega\right)-q_{0} e^{-r_{0} t} \leq z^{N}(t, x, \omega) \leq u\left(t+\zeta_{0}^{+}, x, \omega\right)+q_{0} e^{-r_{0} t}
$$


for all $t \in\left[0, p_{0} N\right]$ where $\zeta_{0}^{ \pm}=\zeta^{ \pm}(0)$ are defined by (4.4) with $\epsilon=q_{0}=\epsilon_{0}$. Taking $\xi_{0}^{+}$larger, if necessary, we have $K_{1}:=\zeta_{0}^{+}-\zeta_{0}^{-} \geq 2^{0}$. This establishes (5.14), (5.15), (5.16), (5.17), (5.19), and (5.20) for $n=0$ and initializes the induction argument. Observe that $\xi_{0}^{ \pm}(\omega)=\xi_{0}^{ \pm}$may be chosen to be deterministic constants, due to the uniform properties of $u$ and the fact that $z_{0}(x)$ is deterministic. So, the initial shifts $\zeta_{0}^{+}$and $\zeta_{0}^{-}$are independent of $\omega \in \Omega$.

Next, we assume (5.14), (5.15), (5.16), (5.17), (5.19), and (5.20) hold for some $n \in[0, \ell N-1]$, and we complete the induction step from $n$ to $n+1 \leq \ell N$. We will consider three regions in the time-space domain: $\Lambda_{R}, \Lambda_{R}^{-}, \Lambda_{R}^{+}$will be the regions near, behind, and before the interface.

1. Near the interface. We first consider the middle region

$$
\Lambda_{R}=\left\{(t, x) \in \mathbb{R}^{2}:\left|x-X\left(t+\left(\zeta_{0}^{+}+\zeta_{0}^{-}\right) / 2\right)\right| \leq R\right\}
$$

with $R>2 L_{0}>0$ chosen sufficiently large so that $\Lambda_{R}$ contains all points $(t, x)$ where both $u\left(t+\zeta_{0}^{-}-1, x\right) \leq\left(1+\theta_{1}\right) / 2$ and $u\left(t+\zeta_{0}^{+}+1, x\right) \geq \theta_{2} / 2$ hold. Due to property (P4) of $u, R$ depends only on the difference $\zeta_{0}^{+}-\zeta_{0}^{-}$. By the induction assumption, $\zeta_{0}^{-}-1 \leq \zeta_{n}^{-} \leq \zeta_{n}^{+} \leq \zeta_{0}^{+}+1$ so that either

$$
u\left(t+\zeta_{n}^{-}, x\right) \geq \frac{\left(1+\theta_{1}\right)}{2} \quad \text { or } \quad u\left(t+\zeta_{n}^{+}, x\right) \leq \frac{\theta_{2}}{2}
$$

holds for all $(t, x) \in \mathbb{R}^{2} \backslash \Lambda_{R}$, for all $n$. If $p_{0}$ is sufficiently small and $N$ is sufficiently large, then

$$
X\left(t+\left(\zeta_{0}^{+}+\zeta_{0}^{-}\right) / 2\right) \leq C_{\max }\left(p_{0} N+\left(\zeta_{0}^{+}+\zeta_{0}^{-}\right) / 2\right) \leq N-3 R
$$

holds for all $t \in\left[0, p_{0} N\right]$. Consequently, $\hat{g}_{N}(x, \omega)=g(x, \omega)$ if $(t, x) \in \Lambda_{3 R}$ and $t \in\left[0, p_{0} N\right]$.

Just as in the case of the ignition type nonlinearity, one may apply the Harnack inequality to conclude that either

$$
u_{n}^{-}\left(t+\gamma\left(\zeta_{n}^{+}-\zeta_{n}^{-}\right), x\right) \leq z^{N}(t, x)
$$

or

$$
u_{n}^{+}\left(t-\gamma\left(\zeta_{n}^{+}-\zeta_{n}^{-}\right), x\right) \geq z^{N}(t, x)
$$

must hold for $(x, t) \in \Lambda_{R}$ and $t \in\left[\bar{s}_{n}+\sigma, \bar{s}_{n}+\sigma+\tau_{2}\right]$ if $\bar{s}_{n}+\sigma+\tau_{2} \leq p_{0} N$. As before, $u_{n}^{-}(t, x)$ and $u_{n}^{+}(t, x)$ denote the functions $u\left(t+\zeta_{n}^{-}, x\right)$ and $u\left(t+\zeta_{n}^{+}, x\right)$, respectively. Also, $\bar{s}_{n}=t_{n}+\tau_{1}$; the parameters $\tau_{1}, \sigma$, and $\tau_{2}$ are chosen as before. We will set $t_{n+1}=\bar{s}_{n}+\sigma+\tau_{2}$, so that $t_{n}=n T$ with $T=\tau_{1}+\sigma+\tau_{2}$ and $\tau_{1}, \sigma$, and $\tau_{2}$ sufficiently large. The condition $\ell \leq p_{0} / T$ implies that $t_{n} \leq p_{0} N$ for all $n \in[0, \ell N]$. Assume for now on that (5.26) holds, rather than (5.27).

2. Behind the interface. This step also may be carried out just as in the case of the ignition-type nonlinearity. We find that

$$
u^{-}\left(t+\gamma\left(\zeta_{n}^{+}-\zeta_{n}^{-}\right), x, \omega\right)-z^{N} \leq e^{-\lambda\left(t-\bar{s}_{n}-\sigma\right)} C\left[2 e^{-r_{2} \sigma}+\gamma\right]\left(\zeta_{n}^{+}-\zeta_{n}^{-}\right)
$$

holds for $(t, x) \in \Lambda_{R}^{-}$, if $t \leq p_{0} N$. Here $\lambda=\min \left(\tau_{0}^{-1} \log 2, \beta\right)$ and

$$
\Lambda_{R}^{-}:=\left\{(t, x): x \leq X\left(t+\left(\zeta_{0}^{+}+\zeta_{0}^{-}\right) / 2\right)-R, \quad t \in\left[\bar{s}_{n}+\sigma, \bar{s}_{n}+\sigma+\tau_{2}\right]\right\} .
$$

3. Ahead of the interface. Finally we consider the region in $\mathbb{R} \backslash \Lambda_{R}$ ahead of interface:

$$
\Lambda_{R}^{+}:=\left\{(t, x): X\left(t+\left(\zeta_{0}^{+}+\zeta_{0}^{-}\right) / 2\right)+R \leq x \leq N, \quad t \in\left[\bar{s}_{n}+\sigma, \bar{s}_{n}+\sigma+\tau_{2}\right]\right\} .
$$

We define the difference $v(t, x)=u_{n}^{-}\left(t+\gamma\left(\zeta_{n}^{+}-\zeta_{n}^{-}\right), x\right)-z^{N}(t, x)$ and bound it from above. Observe that

$$
u_{n}^{-}\left(t+\gamma\left(\zeta_{n}^{+}-\zeta_{n}^{-}\right), x\right) \leq u\left(t+\zeta_{n}^{+}, x\right) \leq u\left(t+\zeta_{0}^{+}+1, x\right) \leq \theta_{2} / 2
$$

in $\Lambda_{R}^{+}$, and

$$
z^{N}(t, x) \leq u\left(t+\zeta_{n}^{+}, x\right)+\left(\zeta_{n}^{+}-\zeta_{n}^{-}\right) e^{-r_{2}\left(t-t_{n}\right)} \leq u\left(t+\zeta_{0}^{+}+1, x\right)+\left(\zeta_{0}^{+}-\zeta_{0}^{-}\right) e^{-r_{2} \sigma} \leq \theta_{2}
$$


if $\sigma>0$ is large enough and $(t, x) \in \Lambda_{R}^{+}$. Therefore, $v$ solves an equation of the form $v_{t}-v_{x x}=$ $a(t, x) v$ with $a(t, x) \leq-\beta<0$ in $\Lambda_{R}^{+}$. Moreover, at the time $t=\bar{s}_{n}+\sigma$ we have

$$
\begin{aligned}
v\left(\bar{s}_{n}+\sigma, x\right) & =u\left(\bar{s}_{n}+\zeta_{n}^{-}+\sigma+\gamma\left(\zeta_{n}^{+}-\zeta_{n}^{-}\right), x\right)-z^{N}\left(\bar{s}_{n}+\sigma, x\right) \\
& \leq q_{n} e^{-r_{2} \sigma}+C \gamma\left(\zeta_{n}^{+}-\zeta_{n}^{-}\right) \\
& \leq C\left[e^{-r_{2} \sigma}+\gamma\right]\left(\zeta_{n}^{+}-\zeta_{n}^{-}\right)
\end{aligned}
$$

for all $x \in \mathbb{R}$. On the left boundary of $\Lambda_{R}^{+}$, the function $v(t, x)$ is non-positive. For $t \in\left[0, p_{0} N\right]$, the right boundary of $\Lambda_{R}^{+}$is where $x=N$ and here

$$
\begin{aligned}
v(t, N) & \leq u\left(t+\zeta_{n}^{-}+\gamma\left(\zeta_{n}^{+}-\zeta_{n}^{-}\right), N\right)-0 \\
& \leq u\left(t+\zeta_{0}^{+}+1, N\right) \\
& \leq C e^{-\left(N-C_{\max }\left(t+\zeta_{0}^{+}+1\right)\right)} \\
& \leq 2^{-N \ell} e^{-\lambda p_{0} N}
\end{aligned}
$$

if $p_{0}$ and $\ell$ are sufficiently small and $N$ is sufficiently large. Thus, $v(t, N) \leq 2^{-n} e^{-\lambda \sigma} e^{-\lambda\left(t-\bar{s}_{n}-\sigma\right)}$ for $t \in\left[0, p_{0} N\right]$ and $n \leq \ell N$.

Applying the comparison principle in the domain $\Lambda_{R}^{+}$, we conclude that

$$
v(t, x) \leq C\left(\zeta_{n}^{+}-\zeta_{n}^{-}\right)\left(e^{-r_{2} \sigma}+\gamma\right) e^{-\beta\left(t-\bar{s}_{n}-\sigma\right)}+2^{-n} e^{-\lambda \sigma} e^{-\lambda\left(t-\bar{s}_{n}-\sigma\right)}
$$

for $(t, x) \in \Lambda_{R}^{+}$and $t \leq p_{0} N$. Using the induction assumption $\left(\zeta_{n}^{+}-\zeta_{n}^{-}\right) \geq 2^{-n}$, so we conclude

$$
v(t, x) \leq C\left(\zeta_{n}^{+}-\zeta_{n}^{-}\right)\left(2 e^{-\lambda \sigma}+\gamma\right) e^{-\lambda\left(t-\bar{s}_{n}-\sigma\right)} .
$$

4. Shrinking the gap $\zeta_{n}^{+}-\zeta_{n}^{-}$. As in [19], we now combine the estimates (5.26), (5.28) and (5.30) and complete the induction step. We have shown that if (5.26) holds (rather than (5.27)) then at times $t \in\left(\bar{s}_{n}+\sigma, \bar{s}_{n}+\sigma+\tau_{2}\right)$ we have

$$
z^{N}(t, x) \geq u\left(t+\zeta_{n}^{-}+\gamma\left(\zeta_{n}^{+}-\zeta_{n}^{-}\right), x\right)
$$

for $(t, x) \in \Lambda_{R}$,

$$
z^{N}(t, x) \geq u\left(t+\zeta_{n}^{-}+\gamma\left(\zeta_{n}^{+}-\zeta_{n}^{-}\right), x\right)-C\left(2 e^{-r_{2} \sigma}+\gamma\right)\left(\zeta_{n}^{+}-\zeta_{n}^{-}\right) e^{-\lambda\left(t-\bar{s}_{n}-\sigma\right)}
$$

for $(t, x) \in \Lambda_{R}^{-}$, and

$$
z^{N}(t, x) \geq u\left(t+\zeta_{n}^{-}+\gamma\left(\zeta_{n}^{+}-\zeta_{n}^{-}\right), x\right)-C\left(2 e^{-\lambda \sigma}+\gamma\right)\left(\zeta_{n}^{+}-\zeta_{n}^{-}\right) e^{-\lambda\left(t-\bar{s}_{n}-\sigma\right)}
$$

for $(t, x) \in \Lambda_{R}^{+}$. We also still have the upper bound:

$$
z^{N}(t, x) \leq u\left(t+\zeta_{n}^{+}, x\right)+q_{n} e^{-r_{2}\left(t-t_{n}\right)}
$$

for all $t \in\left[t_{n}, p_{0} N\right]$ and $x \in(-\infty, N]$, and by the induction assumption $q_{n} \leq\left(\zeta_{n}^{+}-\zeta_{n}^{-}\right)$.

We may now set $t_{n+1}=\bar{s}_{n}+\sigma+\tau_{2}$, so that $t_{n}=n T$ with $T=\tau_{1}+\sigma+\tau_{2}$. We define the new shifts as

$$
\zeta_{n+1 / 2}^{+}=\zeta_{n}^{+}, \quad \zeta_{n+1 / 2}^{-}=\zeta_{n}^{-}+\gamma\left(\zeta_{n}^{+}-\zeta_{n}^{-}\right),
$$

and the new correction $q_{n+1}=C\left(\zeta_{n}^{+}-\zeta_{n}^{-}\right)\left(e^{-r_{2} \sigma}+\gamma\right) e^{-r_{2} \tau_{2}}$. Again, $r_{2}$ is chosen to satisfy $0<r_{2}<\min (\nu, \lambda) \leq \beta$. Then for $t=t_{n+1}$ we conclude that

$$
\begin{aligned}
& z^{N}\left(t_{n+1}, x\right) \geq u\left(t_{n+1}+\zeta_{n+1 / 2}^{-}, x\right)-q_{n+1} \\
& z^{N}\left(t_{n+1}, x\right) \leq u\left(t_{n+1}+\zeta_{n+1 / 2}^{+}, x\right)+q_{n+1} .
\end{aligned}
$$


holds for $x \in(-\infty, N]$. We will complete the induction step by applying Lemma 4.2 to (5.34) and (5.35). To this end, we set

$$
\begin{aligned}
& \zeta_{n+1}^{+}=\zeta_{n+1 / 2}^{+}+K_{3} q_{n+1}=\zeta_{n}^{+}+K_{3}\left(\zeta_{n}^{+}-\zeta_{n}^{-}\right) C\left(e^{-r_{2} \sigma}+\gamma\right) e^{-r_{2} \tau_{2}} \\
& \zeta_{n+1}^{-}=\zeta_{n+1 / 2}^{-}-K_{3} q_{n+1}=\zeta_{n}^{-}+\gamma\left(\zeta_{n}^{+}-\zeta_{n}^{-}\right)-K_{3}\left(\zeta_{n}^{+}-\zeta_{n}^{-}\right) C\left(e^{-r_{2} \sigma}+\gamma\right) e^{-r_{2} \tau_{2}}
\end{aligned}
$$

and $K_{3}=B / r_{0}$.

The bounds (5.16)-(5.19) may be verified just as in the case of the ignition type nonlinearity in Section 4. Since $q_{n+1}=C\left(\zeta_{n+1}^{+}-\zeta_{n+1}^{-}\right) \geq C 2^{-(n+1)}$,

$$
\begin{aligned}
p_{3} \log \left(q_{n+1}\right)-p_{1}+p_{2} N & \geq-p_{3}(n+1) \log 2+\log (C)-p_{1}+p_{2} N \\
& \geq-p_{3} \ell N \log 2+\log (C)-p_{1}+p_{2} N \geq \max \left(0, \zeta_{0}^{+}+1\right) \geq \max \left(0, \zeta_{n+1}^{-}\right)
\end{aligned}
$$

if $\ell$ is sufficiently small and $N$ is sufficiently large. This is (5.20). Observe that the constants defining defining $\sigma, \tau_{1}, \tau_{2}$, and $\gamma$ to not depend on $N$.

Since (5.20) holds for $n+1$, Lemma 5.2 applied to (5.34) and (5.35) implies that for $t \in$ $\left[t_{n+1}, p_{0} N\right]$ we have

$$
\begin{aligned}
& z^{N}(t, x) \geq u\left(t+\zeta_{n+1}^{-}, x\right)-q_{n+1} e^{-r_{2}\left(t-t_{n+1}\right)} \\
& z^{N}(t, x) \leq u\left(t+\zeta_{n+1}^{+}, x\right)+q_{n+1} e^{-r_{2}\left(t-t_{n+1}\right)}
\end{aligned}
$$

for all $x \in(\infty, N]$. This completes the induction and the proof of Proposition 5.3.

Theorem 3.4 now follows directly from Proposition 5.3. Define $\tau^{N}(\omega)$ by

$$
\tau^{N}(\omega)=\left(\zeta_{k}^{-}(\omega)+\zeta_{k}^{+}(\omega)\right) / 2
$$

with $k$ being the largest integer in $[0, \ell N]$. From (5.18) - (5.19) it is easy to see that $\left|\zeta_{n}^{+}-\tau^{N}\right| \leq$ $C e^{-h n}$ for $n \in[0, \ell N]$ and some deterministic constants $C, h>0$ independent of $N$. Therefore, Proposition 5.3 implies that for $t \in\left[t_{n}, t_{n}+1\right]$ and $x \in(-\infty, N]$,

$$
\begin{aligned}
z^{N}(t, x, \omega) & \leq u\left(t+\tau^{N}, x, \omega\right)+M\left(\zeta_{n}^{+}-\tau^{N}\right)+q_{n} e^{-r\left(t-t_{n}\right)} \\
& \leq u\left(t+\tau^{N}, x, \omega\right)+C e^{-r t}
\end{aligned}
$$

A lower bound $z^{N}\left(t, x, \omega \geq u\left(t+\tau^{N}, x, \omega\right)-C e^{-r t}\right.$ for $x \in(-\infty, N]$ also follows from Proposition 5.3. Finally, the bound may be extended to all of $x \in \mathbb{R}$ by using the fact that $z^{N}(t, x, \omega) \leq$ $K e^{-r_{3}\left(N-C_{\max } t\right)}$ for all $x \geq N$. This proves (3.9) and completes the proof of Theorem 3.4.

\section{Proof of Corollary 3.2 and Corollary 3.5}

We prove only Corollary 3.5; the proof of Corollary 3.2 is almost identical and even simpler since the parameter $N$ is not involved. Due to property $(\mathrm{P} 4)$ of the transition front $u(t, x, \omega)$, we may pick $L_{1}>0$ large enough so that both

$$
\begin{aligned}
& u(t, x+X(t, \omega), \omega) \leq \theta_{0} / 3, \quad \forall x>L_{1}, \quad t \in \mathbb{R} \\
& u(t, x+X(t, \omega), \omega) \geq\left(2+\theta_{0}\right) / 3, \quad \forall x<-L_{1}, \quad t \in \mathbb{R}
\end{aligned}
$$

hold with probability one. Then, Theorem 3.4 implies that by choosing $t_{1}$ sufficiently large and $N \geq t_{1} / p_{0}$, we have

$$
\begin{aligned}
& z^{N}(t, x, \omega) \leq \theta_{0} / 2, \quad \forall x>L_{1}+X\left(t+\tau_{N}, \omega\right), \quad t \in\left[t_{1}, p_{0} N\right] \\
& z^{N}(t, x, \omega) \geq\left(1+\theta_{0}\right) / 2, \quad \forall x<-L_{1}+X\left(t+\tau_{N}, \omega\right), \quad t \in\left[t_{1}, p_{0} N\right]
\end{aligned}
$$

with probability one. In the middle region where $\left|x-X\left(t+\tau_{N}, \omega\right)\right| \leq L_{1}$, we have

$$
\begin{aligned}
& z^{N}(t, x, \omega) \leq u\left(t+\tau_{N}, x, \omega\right)+C e^{-r t} \leq u\left(t+\tau_{N}+h, \omega\right)-h \delta_{2 L_{1}}+C e^{-r t} \\
& z^{N}(t, x, \omega) \geq u\left(t+\tau_{N}, x, \omega\right)-C e^{-r t} \geq u\left(t+\tau_{N}-h, \omega\right)+h \delta_{2 L_{1}}-C e^{-r t}
\end{aligned}
$$


for all $t \in\left[0, p_{0} N\right]$, where $\delta_{2 L_{1}}$ is defined by (1.16). Therefore, for $\left|x-X\left(t+\tau_{N}, \omega\right)\right| \leq L_{1}$ and $h \geq C e^{-r t} /\left(\delta_{2 L_{1}}\right)$,

$$
u\left(t+\bar{\tau}_{N}-h, x, \omega\right) \leq z^{N}(t, x, \omega) \leq u\left(t+\tau_{N}+h, x, \omega\right)
$$

holds for all $t \in\left[t_{1}, p_{0} N\right]$, while (6.1) holds away from the interface for $t \in\left[t_{1}, p_{0} N\right]$. Consequently,

$$
X\left(t+\tau_{N}\right)-C_{\max } h \leq X\left(t+\tau_{N}-h\right) \leq X^{N}(t) \leq X\left(t+\tau_{N}+h\right) \leq X\left(t+\tau_{N}\right)+C_{\max } h
$$

for all $h \geq C e^{-r t} /\left(\delta_{2 L_{1}}\right)$. Here $X^{N}(t)$ denotes the interface associated with $z^{N}$ (the largest $x$ such that $\left.z^{N}(t, x, \omega) \geq \theta_{0}\right)$. This implies that

$$
\left|X^{N}(t)-X\left(t+\tau_{N}\right)\right| \leq C_{\max } C \frac{e^{-r t}}{\delta_{2 L_{1}}}
$$

for all $t \in\left[t_{1}, p_{0} N\right]$. Observe that $t_{1}$ is independent of $N$.

Now we are going to choose $t=T_{k}^{N}$ and plug this into (6.3). By (6.3), $X^{N}\left(p_{0} N\right) \geq$ $X\left(p_{0} N+\tau_{N}\right)-C_{\max } C / \delta_{2 L_{1}} \geq C_{\min }\left(p_{0} N-C_{\tau}\right)-C_{\max } C / \delta_{2 L_{1}}$. So if $k \leq k_{N}^{+}:=C_{\min }\left(p_{0} N-\right.$ $\left.C_{\tau}\right)-C_{\max } C / \delta_{2 L_{1}}$, we must have $X^{N}\left(p_{0} N\right) \geq k$ so that $T_{k}^{N} \leq p_{0} N$. Recall that our hypotheses on $z_{0}$ imply that $z^{N}(t, x, \omega) \leq K_{4} e^{-r_{3}\left(x-C_{\max } t\right)}$ for some deterministic constants $K_{4}, r_{3}$. So, if $k \geq k^{-}:=C_{\max } t_{1}+C_{\max }\left(C_{\max } r_{3}\right)^{-1}\left|\log \left(\theta_{0} / K_{4}\right)\right|$, we must have $T_{k}^{N} \geq\left(C_{\max }\right)^{-1} k-$ $\left(C_{\text {max }} r_{3}\right)^{-1}\left|\log \left(\theta_{0} / K_{4}\right)\right| \geq t_{1}$, with probability one. For $N$ sufficiently large, $k^{-}<k_{N}^{+}$. So, if $k \in\left[k^{-}, k_{N}^{+}\right]$, we have $T_{k}^{N} \in\left[t_{1}, p_{0} N\right]$. Consequently, (6.3) applied at $t=T_{k}^{N}$ implies that

$$
k=X^{N}\left(T_{k}^{N}\right) \geq X\left(T_{k}^{N}+\tau_{N}\right)-h
$$

with $h=C_{\max } C e^{-r\left(T_{k}^{N}\right)}$. Therefore, since $X(t)$ is monotone increasing in $t$, we have $T_{k+h} \geq$ $T_{k}^{N}+\tau_{N}$, so that $T_{k}^{N} \leq T_{k+h}-\tau_{N} \leq T_{k}-\tau_{N}+\left(C_{m i n}\right)^{-1} h \leq T_{k}-\tau_{N}+C_{2} e^{-C_{3} k}$. Similarly, (6.3) also implies that

$$
k=X^{N}\left(T_{k}^{N}\right) \leq X\left(T_{k}^{N}+\tau_{N}\right)+h
$$

so that $T_{k-h} \leq T_{k}^{N}+\tau_{N}$. Hence, $T_{k}^{N} \geq T_{k-h}-\tau_{N} \geq T_{k}-\tau_{N}-\left(C_{m i n}\right)^{-1} h \geq T_{k}-\tau_{N}-C_{2} e^{-C_{3} k}$.

Combining these observations, we conclude that for almost every $\omega \in \Omega, \mid T_{k}^{N}(\omega)-T_{k}(\omega)+$ $\tau_{N}(\omega) \mid \leq C_{2} e^{-C_{3} k}$ for $k \in\left[k^{-}, k_{N}^{+}\right]$and some constants $C_{2}, C_{3}>0$. This immediately implies (3.11) with $s=C_{\min } p_{0} / 2$ and $C$ sufficiently large.

\section{An example of Case A}

In this section we construct an example in which Case A of Theorem 1.1 holds. Let us suppose that $f_{0}$ is the ignition nonlinearity. Let $\left\{\xi_{k}(\omega)\right\}_{k \in \mathbb{Z}}$ be independent, identically distributed random variables defined over probability space $(\Omega, \mathcal{F}, \mathbb{P})$. Suppose that $\xi_{k}=1$ or $\xi_{k}=-1$, each with probability $1 / 2$. For $L>0$, let the random variable $r(\omega)$ be uniformly distributed on $[0, L]$ and independent of each $\xi_{k}$. For each $k \in \mathbb{Z}$, define the interval $I_{k}=[L k, L(k+1))$. Define the random field

$$
g_{0}(x, \omega)= \begin{cases}g^{\max }, & \text { if } x-r(\omega) \in I_{k}, \quad \xi_{k}(\omega)=1 \\ g^{\min }, & \text { if } x-r(\omega) \in I_{k}, \quad \xi_{k}(\omega)=-1 .\end{cases}
$$

Let $g(x, \omega)=\phi^{\epsilon} * g_{0}(x, \omega)=\int_{\mathbb{R}} \phi^{\epsilon}(x-y) g_{0}(y, \omega) d y$, where $\phi^{\epsilon}(x) \geq 0$ is a smooth approximation of the identity with compact support in the interval $[-\epsilon, \epsilon], \epsilon \in(0,1)$. The point is that for almost every $\omega \in \Omega, g(x, \omega)$ is constant on large intervals of length $L-2 \epsilon$. On these intervals, the interface will move with average speed approximately equal to either $C_{\max }$ or $C_{\min }$ with equal probability. Since the $\xi_{k}$ are independent, the speed in one interval will be approximately independent of the speed in the next interval, as we now demonstrate. 
We now show that for for some constant $C>0$,

$$
\sigma^{2}=\lim _{n \rightarrow \infty} \frac{1}{n} \mathbb{E}\left[\left|T_{n}(\omega)-n \bar{\tau}\right|^{2}\right] \geq C L>0
$$

if $L$ is sufficiently large. Observe that

$$
T_{(N+1) L}-(N+1) L \bar{\tau}=s^{-}+\left(\sum_{j=0}^{N-1} s_{j}\right)+s^{+}
$$

where $s^{-}=\left(T_{r(\omega)}-r(\omega) \bar{\tau}\right), s_{j}=T_{r(\omega)+(j+1) L}-T_{r(\omega)+j L}-L \bar{\tau}$, and $s^{+}=T_{(N+1) L}-T_{r(\omega)+N L}-$ $(L-r(\omega)) \bar{\tau}$. Hence, by the triangle inequality,

$$
\mathbb{E}\left[\left|T_{(N+1) L}-(N+1) L \bar{\tau}\right|^{2}\right]^{1 / 2} \geq \mathbb{E}\left[\left|\sum_{j=0}^{N-1} s_{j}\right|^{2}\right]^{1 / 2}-\mathbb{E}\left[\left(s^{-}+s^{+}\right)^{2}\right]^{1 / 2} .
$$

Since $s^{-}$and $s^{-}$are both bounded by $L\left(\left(C_{\min }\right)^{-1}+\bar{\tau}\right)$, independently of $N$, we have $\mathbb{E}\left[\left(s^{-}+\right.\right.$ $\left.\left.s^{+}\right)^{2}\right] /((N+1) L) \leq C L /(N+1)$, which vanishes as $N \rightarrow \infty$. Therefore, (7.2) and a similar upper bound, imply

$$
\begin{aligned}
\sigma^{2} & =\lim _{N \rightarrow \infty} \frac{1}{(N+1) L} \mathbb{E}\left[\left|T_{(N+1) L}-(N+1) L \bar{\tau}\right|^{2}\right] \\
& =\lim _{N \rightarrow \infty} \frac{1}{(N+1) L} \mathbb{E}\left[\left|\sum_{j=0}^{N-1} s_{j}\right|^{2}\right]=\frac{1}{L} \mathbb{E}\left[s_{0}^{2}\right]+\frac{2}{L} \sum_{j=1}^{\infty} \mathbb{E}\left[s_{0} s_{j}\right]
\end{aligned}
$$

Define the random variables $\left\{\beta_{j}\right\}_{j \in \mathbb{Z}}$ by

$$
\beta_{j}(\omega)=\left(\frac{1}{C_{\min }}-\bar{\tau}\right) \mathbb{I}_{\xi_{j}<0}+\left(\frac{1}{C_{\max }}-\bar{\tau}\right) \mathbb{I}_{\xi_{j}>0} .
$$

Here $\mathbb{I}_{\xi_{j}<0}$ denotes the indicator function of the set $\left\{\omega \mid \xi_{j}(\omega)<0\right\}$. We claim that there is a finite constant $C>0$, independent of $L$, such that

$$
\left\|s_{j}-L \beta_{j}\right\|_{L^{\infty}(\Omega, \mathbb{P})} \leq C
$$

for all $j \geq 0$. Postponing the proof of this claim, we now show how (7.4) implies $\sigma^{2} \geq C L$ for some constant $C>0$, if $L$ is sufficiently large.

First, observe that $0=\mathbb{E}\left[s_{j}\right]=L \mathbb{E}\left[\beta_{j}\right]+\mathbb{E}\left[s_{j}-L \beta_{j}\right]$, and from (7.4) it follows that

$$
\left|\mathbb{E}\left[\beta_{j}\right]\right| \leq C L^{-1} .
$$

Now for $k \geq j$ we compute

$$
\begin{aligned}
\mathbb{E}\left[s_{j} s_{k}\right]= & \mathbb{E}\left[\left(L \beta_{j}\right)\left(L \beta_{k}\right)\right]+\mathbb{E}\left[\left(s_{j}-L \beta_{j}\right)\left(L \beta_{k}\right)\right] \\
& +\mathbb{E}\left[\left(L \beta_{j}\right)\left(s_{k}-L \beta_{k}\right)\right]+\mathbb{E}\left[\left(s_{j}-L \beta_{j}\right)\left(s_{k}-L \beta_{k}\right)\right] .
\end{aligned}
$$

So, from (7.4) we conclude that

$$
\left|\mathbb{E}\left[s_{j} s_{k}\right]-L^{2} \mathbb{E}\left[\beta_{j} \beta_{k}\right]\right| \leq C L .
$$

For $k \neq j$, we use (7.5) and the independence of the $\xi_{k}$ to obtain $\left|\mathbb{E}\left[\beta_{j} \beta_{k}\right]\right|=\left|\mathbb{E}\left[\beta_{j}\right] \mathbb{E}\left[\beta_{k}\right]\right| \leq C L^{-2}$. This and (7.6) implies that for any $j \neq k$,

$$
\left|\mathbb{E}\left[s_{j} s_{k}\right]\right| \leq C L
$$

Since $C_{\max }>C_{\min }$, we observe that $\mathbb{E}\left[\beta_{j}^{2}\right]>\epsilon_{1}$ for some constant $\epsilon_{1}>0$. 
Now we also know that for any integer $k$, and $1 \leq k \leq j$,

$$
\mathbb{E}\left[s_{0} s_{j}\right]=\mathbb{E}\left[\left(H_{L}^{-} s_{0}\right)\left(H_{k L}^{+} s_{j}\right)\right]+\mathbb{E}\left[\left(H_{L}^{-} s_{0}\right)\left(\left(I-H_{k L}^{+}\right) s_{j}\right)\right],
$$

where $H_{k}^{ \pm}$denote the projections $H_{k}^{ \pm} \theta=\mathbb{E}\left[\theta \mid \hat{\mathcal{F}}_{k}^{ \pm}\right]$, where $\hat{\mathcal{F}}_{k}^{+}$and $\hat{\mathcal{F}}_{k}^{+}$are the $\sigma$-algebras generated by $\left\{\xi_{j}\right\}_{j \leq k}$ and $\left\{\xi_{j}\right\}_{j>k}$, respectively (which are independent of $r(\omega)$ ). By Proposition 2.2 it follows that

$$
\begin{aligned}
\left\|\left(I-H_{k L}^{+}\right) s_{j}\right\|_{L^{2}(\Omega, \mathbb{P})} & =\left\|\sum_{n=0}^{L-1}\left(I-H_{k L}^{+}\right) \Delta T_{r(\omega)+j L+n}\right\|_{L^{2}(\Omega, \mathbb{P})} \\
& \leq \sum_{n=0}^{L-1} C_{1} e^{-C_{2}(j L+n-k L)} \leq C e^{-C_{2} L(j-k)} .
\end{aligned}
$$

Since the $\sigma$-algebras $\hat{\mathcal{F}}_{L}^{-}$and $\hat{\mathcal{F}}_{k L}^{+}$are independent for $k>1, \mathbb{E}\left[\left(H_{L}^{-} s_{0}\right)\left(H_{k L}^{+} s_{j}\right)\right]=0$, so that

$$
\left|\mathbb{E}\left[s_{0} s_{j}\right]\right| \leq\left\|s_{0}\right\|_{L^{2}(\Omega, \mathbb{P})}\left\|\left(I-H_{2 L}^{+}\right) s_{j}\right\|_{L^{2}(\Omega, \mathbb{P})} \leq L^{2} C e^{-C_{2} L(j-2)}
$$

holds for $j \geq 2$.

Finally, we combine (7.7), (7.10),

$$
\begin{aligned}
\sigma^{2}=\frac{1}{L} \mathbb{E}\left[s_{0}^{2}\right]+\frac{2}{L} \sum_{j=1}^{\infty} \mathbb{E}\left[s_{0} s_{j}\right] & \geq \frac{1}{L}\left(\epsilon_{1} L^{2}+2 \sum_{j=1}^{R} \mathbb{E}\left[s_{0} s_{j}\right]+2 \sum_{j=R+1}^{\infty} \mathbb{E}\left[s_{0} s_{j}\right]\right) \\
& \geq \frac{1}{L}\left(\epsilon_{1} L^{2}-R C L-\sum_{j=R+1}^{\infty} L^{2} C e^{-C_{2} L(j-2)}\right)
\end{aligned}
$$

Thus, by first letting $R$ be sufficiently large, and then letting $L$ be sufficiently large, we conclude that $\sigma^{2} \geq \epsilon_{1} L / 2>0$ for all $L$ sufficiently large.

It remains to establish the claim (7.4). Since the $\left\{\xi_{j}\right\}_{j \in \mathbb{Z}}$ are identically distributed, it suffices to prove the claim for $j=0$. The claim will follow from Theorem 3.1 and Corollary 3.2. Let $\phi^{+}(t, x)$ and $\phi^{-}(t, x)$ be the unique traveling wave solutions to $\phi_{t}=\phi_{x x}+g^{\max } f_{0}(\phi)$ and $\phi_{t}=$ $\phi_{x x}+g^{\min } f_{0}(\phi)$, normalized by $\phi(0,0)=\theta_{0}$. These waves move with speed $C_{\max }$ and $C_{\text {min }}$ respectively. Then define

$$
z(t, x, \omega)= \begin{cases}\phi^{+}(t, x), & \text { if } \xi_{0}(\omega)>0 \\ \phi^{-}(t, x), & \text { if } \xi_{0}(\omega)<0\end{cases}
$$

and

$$
\hat{g}(\omega)= \begin{cases}g^{\max }, & \text { if } \xi_{0}(\omega)>0 \\ g^{\min }, & \text { if } \xi_{0}(\omega)<0\end{cases}
$$

For $x \in[r(\omega)+\epsilon, r(\omega)+L-\epsilon], g(x, \omega)=\hat{g}(\omega)$. Also, for $t \leq T_{r(\omega)+L-1}(\omega)$, we have $X(t, \omega) \leq$ $r(\omega)+L-1 \leq r(\omega)+L-\epsilon$, so $z(t, x, \omega)$ and $u(t, x, \omega)$ both satisfy

$$
\begin{aligned}
& z_{t}=z_{x x}+\hat{g}(\omega) f_{0}(z) \\
& u_{t}=u_{x x}+\hat{g}(\omega) f_{0}(u)
\end{aligned}
$$

for all $x \in[r(\omega)+\epsilon, \infty), t \leq T_{r(\omega)+L-1}(\omega)$. For $x \geq r(\omega)+L-\epsilon,(7.15)$ holds because $u \leq \theta_{0}$ for all $x \geq r(\omega)+L-1$ and $t \leq T_{r(\omega)+L-1}(\omega)$, so that $f_{0}(u)=0$ in this region. Now, it follows from Theorem 3.1 that

$$
\sup _{x \in \mathbb{R}}|z(t, x-r(\omega), \omega)-u(t+\tau(\omega), x, \omega)| \leq C e^{-r t}
$$


holds for $t \leq T_{r(\omega)+L-1}(\omega)$. Then, from Corollary 3.2, we have

$$
\begin{aligned}
\left\|T_{L-1}^{z}-\left(T_{r(\omega)+L-1}-T_{r(\omega)}\right)\right\|_{L^{\infty}(\Omega, \mathbb{P})} & =\left\|\sum_{k=0}^{L-2} \Delta T_{k}^{z}-\left(T_{r(\omega)+k+1}-T_{r(\omega)+k}\right)\right\|_{L^{\infty}(\Omega, \mathbb{P})} \\
& \leq \sum_{k=0}^{L-2} C e^{-h k}
\end{aligned}
$$

which is bounded, independently of $L$. However, by construction,

$$
T_{L-1}^{z}(\omega)=(L-1)\left(\frac{1}{C_{\min }} \mathbb{I}_{\xi_{0}<0}+\frac{1}{C_{\max }} \mathbb{I}_{\xi_{0}>0}\right)=(L-1)\left(\beta_{0}+\bar{\tau}\right)
$$

Therefore,

$$
\begin{aligned}
\left\|s_{0}-L \beta_{0}\right\|_{L^{\infty}} & =\left\|T_{r(\omega)+L}-T_{r(\omega)}-L \bar{\tau}-L \beta_{0}\right\|_{L^{\infty}} \\
& =\left\|\left(T_{r(\omega)+L}-T_{r(\omega)+L-1}\right)+\left(T_{r(\omega)+L-1}-T_{r(\omega)}\right)-L\left(\beta_{0}+\bar{\tau}\right)\right\|_{L^{\infty}} \\
& \leq\left(C_{\text {min }}\right)^{-1}+\left\|T_{L-1}^{z}-\left(T_{r(\omega)+L-1}-T_{r(\omega)}\right)\right\|_{L^{\infty}}+\left(C_{\text {min }}\right)^{-1} .
\end{aligned}
$$

Due to (7.17), this establishes the claim (7.4).

In view of (1.26) the above analysis shows that $\kappa^{2}=\left(c^{*}\right)^{3} \sigma^{2} \geq\left(c^{*}\right)^{3} C L$, for $L$ sufficiently large. The asymptotic speed $c^{*}$ also depends on $L$, and in this example it is easy to use (7.1) and (7.4) to show that

$$
\lim _{L \rightarrow \infty} c^{*}(L)=\lim _{L \rightarrow \infty} \bar{\tau}^{-1}=2\left(\frac{1}{C_{\min }}+\frac{1}{C_{\max }}\right)^{-1} .
$$

\section{References}

[1] D.G. Aronson and H.F. Weinberger, Nonlinear diffusion in population genetics, combustion, and nerve propagation, in Partial Differential Equations and Related Topics. J.A. Goldstein, ed. Lecture Notes in Mathematics 446, pp. 5-49, New York: Springer 1975.

[2] H. Berestycki and F. Hamel, Front propagation in periodic excitable media, Comm. Pure Appl. Math. 55 (2002), pp. 949-1032.

[3] H. Berestycki and F. Hamel, Generalized travelling waves for reaction-diffusion equations, In: Perspectives in Nonlinear Partial Differential Equations. In honor of H. Brezis, Contemp. Math. 446 (2007), Amer. Math. Soc., pp. 101-123.

[4] H. Berestycki, F. Hamel, H. Matano, Bistable traveling waves around an obstacle, Comm. Pure Appl. Math 62 (2009), pp. 729-788.

[5] P. Billingsley, "Convergence of Probability Measures." John Wiley and Sons, New York, 1968.

[6] P.C. Fife and J.B. McLeod, The approach of solutions of nonlinear diffusion equations by travelling front solutions, Arch. Rat. Mech. Anal., 65 (1977), pp. 335-361.

[7] R. Fisher, The wave of advance of advantageous genes, Ann. Eugenics, 7 (1937), 355-369.

[8] M. Freidlin, Functional Integration and Partial Differential Equations. Ann. Math. Stud. 109, Princeton University Press, Princeton, NJ, 1985.

[9] J. Gärtner and M.I. Freidlin, The propagation of concentration waves in periodic and random media, Dokl. Acad. Nauk SSSR, 249 (1979), pp. 521-525.

[10] M.I. Gordin, The central limit theorem for stationary processes, Soviet Math. Dokl. 10 (1969) 1174-1176.

[11] P. Hall and C.C. Heyde, Martingale limit Theory and its Application, Academic Press, New York: 1980. 
[12] Ja. Kanel, Stabilization of the solutions of the equations of combustion theory with finite initial functions. (Russian) Mat. Sb. (N.S.) 65 (107), 1964, pp. 398-413.

[13] Ja. Kanel, Stabilization of solutions of the Cauchy problem for certain linear parabolic equations. (Russian) Uspehi Mat. Nauk 18, (1963) (110), pp. 127-134.

[14] Ja. Kanel, Stabilization of solutions of the Cauchy problem for equations encountered in combustion theory. (Russian) Mat. Sb. (N.S.) 59 (101), (1962) suppl., pp. 245-288.

[15] H. Kesten, G. Papanicolaou A limit theorem for turbulent diffusion. Comm. Math. Phys. 65 (1979), pp. 97-128.

[16] A.N. Kolmogorov, I.G. Petrovskii and N.S. Piskunov, Étude de l'équation de la chaleurde matiére et son application à un problème biologique, Bull. Moskov. Gos. Univ. Mat. Mekh. 1 (1937), 1-25.

[17] T.J. Lewis and J.P. Keener, Wave-block in excitable media due to regions of depressed excitability, SIAM J. Appl. Math. 61, 2000, pp.293-316.

[18] H. Matano, A lecture presented at Institut Henri Poincaré, Paris, September, 2002.

[19] A. Mellet, J. Nolen, L. Ryzhik, J.-M. Roquejoffre, Stability of Generalized Transition Fronts. Communications in PDE, accepted for publication, Comm. PDE, 34 (2009), pp. 521-552.

[20] A. Mellet, J.-M. Roquejoffre and Y. Sire, Generalized travelling fronts for local or nonlocal reaction-diffusion equations, Discret Cont. Dyn. Syst. 26 (2010), pp. 303-312.

[21] J. Nolen and L. Ryzhik, Traveling waves in a one-dimensional heterogeneous medium. AIHPAnalyse Nonlinéaire 26 (2009), pp. 1021-1047.

[22] F. Rezakhanlou, Central limit theorem for stochastic Hamilton-Jacobi equation, Comm. Math. Phys. 211 (2000), pp. 413-438.

[23] F. Rothe, Convergence to pushed fronts, Rocky Mountain J. Math. 11 (1981), pp. 617-633.

[24] W. Shen, Traveling waves in diffusive random media, J. Dynamics and Diff. Eqns., 16 (2004), pp. 1011 - 1060.

[25] N. Shigesada, K. Kawasaki, E. Teramoto, Traveling periodic waves in heterogeneous environments. Theoret. Population Biol. 30 (1986), pp. 143-160.

[26] D. Volný, Approximating martingales and the central limit theorem for strictly stationary processes, Stoch. Processes Appls. 44 (1993), pp. 41-74.

[27] J. Wehr and J. Xin, White noise perturbation of the viscous shock fronts of the Burgers equation, Comm. Math. Phys. 181 (1996), pp. 183-203.

[28] J. Wehr and J. Xin, Front speed in the Burgers equation with a random flux, J. Stat. Phys. 88 (1997), pp. 843-871.

[29] S.R.S. Varadhan and N. Zygouras, Behavior of the solution of a random semilinear heat equation, Comm. Pure Appl. Math. 61 (2008), pp. 1298-1329.

[30] J. Xin, Analysis and modelling of front propagation in heterogeneous media, SIAM Rev., 42 (2000), pp. 161-230.

[31] A. Zlatǒs. Generalized traveling waves in disordered media: Existence, uniqueness, and stability, preprint 2010. 